\title{
An Algorithmic Survey of Parametric Value Function Approximation
}

\author{
Matthieu Geist and Olivier Pietquin (Senior Member, IEEE)
}

\begin{abstract}
Reinforcement learning is a machine learning answer to the optimal control problem. It consists in learning an optimal control policy through interactions with the system to be controlled, the quality of this policy being quantified by the so-called value function. A recurrent subtopic of reinforcement learning is to compute an approximation of this value function when the system is too large for an exact representation. This survey reviews state-of-the-art methods for (parametric) value function approximation by grouping them into three main categories: bootstrapping, residual and projected fixed-point approaches. Related algorithms are derived by considering one of the associated cost functions and a specific minimization method, generally a stochastic gradient descent or a recursive least-squares approach.
\end{abstract}

Index Terms - Reinforcement learning, survey, value function approximation

\section{INTRODUCTION}

Reinforcement Learning (RL) [1][2][3][4][5][6][7] addresses the problem of the optimal control of a stochastic dynamic system. In this paradigm, an artificial agent learns an optimal control policy through interactions with the dynamic system (also considered as its environment). After each interaction, the agent receives an immediate scalar reward information and the optimal policy it searches for is the one that maximizes the expected cumulative reward over the long term. The system to be controlled is usually modeled as a Markovian Decision Process (MDP). An MDP is made up of a set of states (the different configurations of the system), a set of actions (which cause a change of the system's state), a set of Markovian transition probabilities (the probability to transit from one state to another under a given action; the Markovian property states that the probability depends on the current state-action pair and not on the path followed to reach it), a reward function associating a scalar to each transition and a discounting factor which decreases long-term rewards' influence. How the agent acts with the system is modeled by a policy which associates to each state a probability distribution over actions. The quality of such a policy is quantified by a value function which associates to each state the expected cumulative discounted reward from starting in the considered state and then following the given policy (expectation being done over all possible trajectories). An optimal policy is

Matthieu Geist and Olivier Pietquin are with the IMS-MaLIS Research Group, Supélec (France). Olivier Pietquin is also with the UMI 2958 (GeorgiaTech - CNRS). one of those which maximize the associated value function for each state.

Thanks to the Markovian property, value functions can be (more or less easily) computed using Bellman equations. The value function of a given policy satisfies the (linear) Bellman evaluation equation and the optimal value function (which is linked to one of the optimal policies) satisfies the (nonlinear) Bellman optimality equation. These Bellman equations are very important for reinforcement learning, as they allow computing the value function.

If the model (that is transition probabilities and the reward function) is known and if state and action spaces are small enough, the optimal policy can be computed using dynamic programming. A first scheme, called policy iteration, consists in evaluating an initial policy (that is computing the associated value function using the linear Bellman evaluation equation) and then improving this policy, the new one being greedy with respect to the computed value function (it associates to each state the action which maximizes the expected cumulative reward obtained from starting in this state, applying this action and then following the initial policy). Evaluation and improvement steps are iterated until convergence (which occurs in a finite number of iterations). A second scheme, called value iteration, consists in computing directly the optimal value function (using the nonlinear Bellman optimality equation and an iterative scheme based on the fact that the value function is the unique fixed-point of the associated Bellman operator). The optimal policy is greedy with respect to the optimal value function.

Reinforcement learning aims at estimating the optimal policy without knowing the model and from interactions with the system. Value functions can no longer be exactly computed, they have to be estimated, which is the main scope of this paper. Reinforcement learning heavily relies on dynamic programming, in the sense that most approaches are some sorts of generalization of value or policy iteration. A first problem is that computing a greedy policy (required for both schemes) from a value function requires the model to be known. The action-value (or Q-) function alleviates this problem by encoding the value of state-action couples (instead of states only). It is defined, for a given policy and for a state-action couple, as the expected discounted cumulative reward starting in the given state, applying the given action and then following the fixed policy. A greedy policy can thus be obtained by maximizing the Q-function over actions.

There are two main approaches to estimate an optimal 
policy through value functions. The first one, based on value iteration, consists in estimating directly the optimal action-value function which is then used to derive an estimate of the optimal policy (with the drawback that errors in the Q-function estimation can lead to a bad derived policy). The second one, based on policy iteration, consists in mixing the estimation of the Q-function of the current policy (policy evaluation) with policy improvement in a generalized policy iteration scheme (generalized in the sense that evaluation and improvement processes interact, independently of the granularity and other details). This scheme presents many variations. Generally, the Qfunction is not perfectly estimated when the improvement step occurs (optimistic policy iteration). Each change in the policy implies a change in the associated Q-function; therefore, the estimation process can be non-stationary. The control policy can be derived from the estimated action-value function (for example, using a Boltzmann distribution or an $\epsilon$-greedy policy). There is generally an underlying dilemma between exploration and exploitation. At each time step, the agent should decide between acting greedily with respect to its uncertain and imperfect knowledge of the world (exploitation) and taking another action which improves this knowledge and possibly leads to a better policy (exploration). The policy can also have its own representation, which leads to actor-critic architectures (which are a form of policy iteration). The actor is the policy, and the critic is an estimated value or Qfunction which is used to correct the policy representation.

All these approaches share a common subproblem: estimating the (action-) value function, of a given policy (evaluation operator) or the optimal one (optimality operator) directly. This issue is even more involved when state or action spaces are too large for a tabular representation, which implies to use some approximate representation. Generally speaking, estimating a function from samples is addressed by the supervised learning paradigm. However, in reinforcement learning, the (action-) values are not directly observed, which renders the underlying estimation problem more difficult. Despite this, a number of (action-) value function estimation algorithms have been proposed in the past decades. The aim of this paper is to review a panel as large as possible of these algorithms by adopting a unifying view which classifies them into three main categories: bootstrapping approaches (Sec. III), residual approaches (Sec. IV) and projected fixed-point approaches (Sec. V). Each of them is related to a specific cost function, and algorithms are derived considering one of these costs and a specific way to minimize it (almost always a stochastic gradient descent or a recursive least-squares approach). Sections III to V are the core of this survey on parametric value function approximation, but additional important topics are briefly covered at the end of the article. Sec. VI shows how these approaches can be embedded in the more general control problem, Sec. VII discusses when choosing what algorithm (among surveyed methods and depending on the practical context) and Sec. VIII provides a brief overview of other ways to estimate a value function, not covered here (such as nonparametric approaches or extensions to eligibility traces). Before this, the underlying formalism is presented in Sec. II.

\section{Preliminaries}

A Markovian decision process (MDP) is a tuple $\{S, A, P, R, \gamma\}$ where $S$ is the (finite) state space, $A$ the (finite) action space, $P: s, a \in S \times A \rightarrow p(. \mid s, a) \in$ $\mathcal{P}(S)$ the family of Markovian transition probabilities, $R: s, a, s^{\prime} \in S \times A \times S \rightarrow r=R\left(s, a, s^{\prime}\right) \in \mathbb{R}$ the bounded deterministic reward function and $\gamma$ the discount factor weighting long term rewards. According to these definitions, the system stochastically steps from state to state conditionally to the actions the agent performed. Let $i$ be the discrete time step. To each transition $\left(s_{i}, a_{i}, s_{i+1}\right)$ is associated an immediate reward $r_{i}$. The action selection process is driven by a policy $\pi: s \in S \rightarrow \pi(. \mid s) \in \mathcal{P}(A)$.

\section{A. Bellman Equations}

The quality of a policy is quantified by the value function $V^{\pi} \in \mathbb{R}^{S}$, defined as the expected discounted cumulative reward starting in a state $s$ and then following the policy $\pi$ :

$$
V^{\pi}(s)=\mathbb{E}\left[\sum_{i=0}^{\infty} \gamma^{i} r_{i} \mid s_{0}=s, \pi\right] .
$$

Thanks to the Markovian property, the value function of a policy $\pi$ satisfies the linear Bellman evaluation equation ${ }^{1}$ :

$$
V^{\pi}(s)=\mathbb{E}_{s^{\prime}, a \mid s, \pi}\left[R\left(s, a, s^{\prime}\right)+\gamma V^{\pi}\left(s^{\prime}\right)\right] .
$$

Let us define the Bellman evaluation operator $T^{\pi}: V \in$ $\mathbb{R}^{S} \rightarrow T^{\pi} V \in \mathbb{R}^{S}$ :

$$
\left[T^{\pi} V\right](s)=\mathbb{E}_{s^{\prime}, a \mid s, \pi}\left[R\left(s, a, s^{\prime}\right)+\gamma V\left(s^{\prime}\right)\right] .
$$

The operator $T^{\pi}$ is a contraction and $V^{\pi}$ is its unique fixed-point:

$$
V^{\pi}=T^{\pi} V^{\pi}
$$

An optimal policy $\pi^{*}$ maximizes the associated value function for each state: $\pi^{*} \in \operatorname{argmax}_{\pi \in \mathcal{P}(A)^{S}} V^{\pi}$. The associated optimal value function, written $V^{*}$, satisfies the nonlinear Bellman optimality equation:

$$
V^{*}(s)=\max _{a \in A} \mathbb{E}_{s^{\prime} \mid s, a}\left[R\left(s, a, s^{\prime}\right)+\gamma V^{*}\left(s^{\prime}\right)\right] .
$$

The optimal value function is unique, but this is not necessarily the case for the optimal policy. Let us define the Bellman optimality operator $T^{*}: V \in \mathbb{R}^{S} \rightarrow T^{*} V \in \mathbb{R}^{S}$ :

$$
\left[T^{*} V\right](s)=\max _{a \in A} \mathbb{E}_{s^{\prime} \mid s, a}\left[R\left(s, a, s^{\prime}\right)+\gamma V\left(s^{\prime}\right)\right] .
$$

The operator $T^{*}$ is a contraction and $V^{*}$ is its unique fixed-point:

$$
V^{*}=T^{*} V^{*} .
$$

The action-value (or Q-) function encodes the values of state-action couples. This is useful in a model-free context.

\footnotetext{
${ }^{1} \mathbb{E}_{x_{1} \mid x_{2}}$ denotes the expectation according to random variable $x_{1}$
} conditioned on $x_{2}$ 
It is defined as the expected cumulative reward starting in $s$, applying $a$ and then following $\pi$ :

$$
Q^{\pi}(s, a)=\mathbb{E}\left[\sum_{i=0}^{\infty} \gamma^{i} r_{i} \mid s_{0}=s, a_{0}=a, \pi\right] .
$$

The action-value function $Q^{\pi}$ also satisfies the linear Bellman evaluation equation:

$$
Q^{\pi}(s, a)=\mathbb{E}_{s^{\prime}, a^{\prime} \mid s, a, \pi}\left[R\left(s, a, s^{\prime}\right)+\gamma Q^{\pi}\left(s^{\prime}, a^{\prime}\right)\right] .
$$

It is clear that value and action-value functions are directly linked:

$$
V^{\pi}(s)=\mathbb{E}_{a \mid s, \pi}\left[Q^{\pi}(s, a)\right] .
$$

A Bellman evaluation operator related to the Q-function can also be defined. By a slight abuse of notation, it is also written $T^{\pi}$, the distinction being clear from the context $\left(T^{\pi}: Q \in \mathbb{R}^{S \times A} \rightarrow T^{\pi} Q \in \mathbb{R}^{S \times A}\right):$

$$
\left[T^{\pi} Q\right](s, a)=\mathbb{E}_{s^{\prime}, a^{\prime} \mid s, a, \pi}\left[R\left(s, a, s^{\prime}\right)+\gamma Q\left(s^{\prime}, a^{\prime}\right)\right] .
$$

This operator is also a contraction and $Q^{\pi}$ is its unique fixed-point:

$$
Q^{\pi}=T^{\pi} Q^{\pi}
$$

The optimal action-value function $Q^{*}$ satisfies the nonlinear Bellman optimality equation too:

$$
Q^{*}(s, a)=\mathbb{E}_{s^{\prime} \mid s, a}\left[R\left(s, a, s^{\prime}\right)+\gamma \max _{a^{\prime} \in A} Q^{*}\left(s^{\prime}, a^{\prime}\right)\right] .
$$

The associated Bellman optimality operator is defined as (with the same slight abuse of notation) $T^{*}: Q \in \mathbb{R}^{S \times A} \rightarrow$ $T^{*} Q \in \mathbb{R}^{S \times A}$ :

$$
\left[T^{*} Q\right](s, a)=\mathbb{E}_{s^{\prime} \mid s, a}\left[R\left(s, a, s^{\prime}\right)+\gamma \max _{a^{\prime} \in A} Q\left(s^{\prime}, a^{\prime}\right)\right] .
$$

This is still a contraction and $Q^{*}$ is its unique fixed-point:

$$
Q^{*}=T^{*} Q^{*}
$$

\section{B. Relaxed Assumptions}

As mentioned in Sec. I, an important subtopic of reinforcement learning is to estimate the (action-) value function of a given policy or directly the Q-function of the optimal policy (in all cases the fixed-point of a Bellman operator) from samples, that is observed trajectories of actual interactions. More precisely, learning is done from a trajectory sampled according to a policy $\pi$, which can be seen as a set of transitions:

$$
\left\{\left(s_{j}, a_{j}, r_{j}, s_{j+1}, a_{j+1}\right)_{1 \leq j \leq i}\right\} .
$$

A first problem is that the model (that is transition probabilities and reward function) is assumed to be unknown. Therefore, no Bellman operator (from which we search a fixed-point for) can be computed. However, they can be estimated from transitions. The sampled Bellman value function evaluation operator $\hat{T}_{j}^{\pi}$, to be linked to Eq. (1), is defined for a transition $\left(s_{j}, r_{j}, s_{j+1}\right)$ as:

$$
\hat{T}_{j}^{\pi}: V \in \mathbb{R}^{S} \rightarrow \hat{T}_{j}^{\pi} V \in \mathbb{R}: \hat{T}_{j}^{\pi} V=r_{j}+\gamma V\left(s_{j+1}\right) .
$$

Notice that a sampled Bellman optimality operator cannot be defined for the value function (to be linked to Eq. (2)), as the maximum depends on the expectation. Similarly, a sampled Bellman evaluation operator can be defined for the Q-function. For a transition $\left(s_{j}, a_{j}, r_{j}, s_{j+1}, a_{j+1}\right)$, it is defined as:

$\hat{T}_{j}^{\pi}: Q \in \mathbb{R}^{S \times A} \rightarrow \hat{T}_{j}^{\pi} Q \in \mathbb{R}: \hat{T}_{j}^{\pi} Q=r_{j}+\gamma Q\left(s_{j+1}, a_{j+1}\right)$.

Last but not least, a sampled Bellman optimality operator can be defined for the Q-function (thanks to the fact that the maximum depends on the expectation and not the contrary). For a transition $\left(s_{j}, a_{j}, r_{j}, s_{j+1}\right)$, we define:

$\hat{T}_{j}^{*}: Q \in \mathbb{R}^{S \times A} \rightarrow \hat{T}_{j}^{*} Q \in \mathbb{R}: \hat{T}_{j}^{*} Q=r_{j}+\gamma \max _{a \in A} Q\left(s_{j+1}, a\right)$.

A second problem is that the state space is usually too large to allow an exact representation of the (action-) value function. Therefore, an approximate representation should be adopted. This article focuses on parametric approaches: the estimated value (respectively action-value) function is of the form $\hat{V}_{\theta}$ (respectively $\hat{Q}_{\theta}$ ), where $\theta$ is the parameter vector; this estimate belongs to an hypothesis space,

$$
\mathcal{H}=\left\{\hat{V}_{\theta}\left(\operatorname{resp} . \hat{Q}_{\theta}\right) \mid \theta \in \mathbb{R}^{p}\right\},
$$

which specifies the architecture of the approximation. For example, if the state space is sufficiently small an exact tabular representation can be chosen for the value function. The estimate is thus of the form $\hat{V}_{\theta}(s)=e_{s}^{T} \theta$ with $e_{s}$ being an unitary vector which is equal to one in the component corresponding to state $s$ and zero elsewhere. A common choice in RL is to adopt a linearly parameterized value function: in this case, $\hat{V}_{\theta}(s)=\phi(s)^{T} \theta$ with $\phi(s)$ a vector of basis functions (e.g., a linear radial basis function network) and $\theta$ the set of associated weights. More complex hypothesis spaces can be envisioned, such as neural networks. However, notice that some of the approaches reviewed in this paper do not allow handling nonlinear representations.

\section{Survey Overview}

Estimating a function from samples is a common topic of supervised learning. However, estimating a value function is a harder problem. Indeed, values are never directly observed, so it is not a regression problem. The available information is provided by the gathered rewards, of which the accumulation defines the value function. Therefore, supervised learning techniques cannot be directly applied to learn such a function ${ }^{2}$. This article reviews state-ofthe-art (parametric) value function estimation algorithms by grouping them into three categories. It will be shown that all approaches minimize the following empirical cost

\footnotetext{
${ }^{2}$ Actually, this is not quite true. One can perform Monte Carlo rollouts starting from a given set of states to obtain related estimated returns (that is estimates of the expected discounted cumulative reward for each state of the set) and use this in any supervised learning scheme. However, this would require being able to sample many possibly infinite trajectories for each state of the set, which is therefore not really practical.
} 
function (given for the Q-function, the value function being a special case):

$$
\theta_{i}=\underset{\omega \in \mathbb{R}^{p}}{\operatorname{argmin}} \sum_{j=1}^{i}\left(\hat{T}_{j} \hat{Q}_{\xi}-\hat{Q}_{\omega}\left(s_{j}, a_{j}\right)\right)^{2}
$$

where $\hat{T}_{j}$ denotes either $\hat{T}_{j}^{\pi}$ or $\hat{T}_{j}^{*}$. Each of the three main approaches is obtained by instantiating the parameter vector $\xi \in \mathbb{R}^{p}$, as explained below and summarized in Tab. I.

a) Bootstrapping approaches $\left(\xi=\theta_{j-1}\right)$ : bootstrapping approaches (reviewed in Sec. III) consist in treating value function approximation as a supervised learning problem and to derive an online algorithm. As values are not directly observable, they are replaced by an estimate computed using a sampled Bellman operator (bootstrapping refers to replacing an unobserved value by an estimate). As first noticed in [8], this corresponds to minimizing cost function (5) instantiated with $\xi=\theta_{j-1}$. If it is minimized using a stochastic gradient descent, this provides TD-Q and Q-learning algorithms [2], given that the sampled Bellman evaluation or optimality operator is considered. Using a (linear) recursive least-squares approach, this gives the fixed-point Kalman filter algorithm [8].

b) Residual approaches $(\xi=\omega)$ : residual approaches (reviewed in Sec. IV) consist in minimizing the squared error between the (action-) value function and its image through a Bellman operator. Practically, a sampled operator is used, which leads to biased estimates. This corresponds to minimizing cost function (5) instantiated with $\xi=\omega$. If it is minimized using a stochastic gradient descent, it provides the residual algorithms of [9]. With a linear recursive least-squares approach, it is the parametric Gaussian Process Temporal Differences algorithm [10], which can be extended using a statistical linearization approach to nonlinear parameterizations and to the optimality operator (the Kalman Temporal Differences framework [11]).

c) Projected fixed-point approaches $\left(\xi=\theta_{i-1} / \theta_{i}\right)$ : projected fixed-point approaches (reviewed in Sec. V) minimize the squared error between the (action-) value function and the image of this function under the (sampled) Bellman operator projected onto the hypothesis space. This is illustrated by Fig. 1 on Page 11. It can be seen as searching for the fixed-point of an operator defined as the composition of the projection with one of the Bellman operators. Computing directly this fixedpoint corresponds to minimizing cost function (5) instantiated with $\xi=\theta_{i}$. Solved using a linear least-squares approach, it gives the Least-Squares Temporal Differences algorithm [12] (generalized using a statistical linearization approach in [13]). Solved using a stochastic gradient descent approach, it provides a bunch of algorithms: Gradient Temporal Difference 2 and Temporal Difference with gradient Correction [14], nonlinear extension of these algorithms [15], as well as $\mathrm{GQ}(\lambda)$, an extension to offpolicy learning and eligibility traces [16], and Greedy-
GQ, an extension to the Bellman optimality operator [17]. Computing the fixed-point associated to the composed operator in an iterative way corresponds to minimizing cost function (5) instantiated with $\xi=\theta_{i-1}$. If solved using a linear least-squares approach, it is the LeastSquares Policy Evaluation algorithm [18], extended to the Bellman optimality operator in [19]. In a batch setting and using any supervised learning algorithm instead of the projection, it is the fitted-Q approach [20][21].

Sec. III to V focus on how one can learn the action-value function from samples. How these samples are generated depends on the way the value function approximator is embedded in a more general control scheme (the ultimate goal of RL being to estimate an optimal policy), which is discussed in Sec. VI.

\section{Bootstrapping Approaches}

Bootstrapping approaches deal with (action-) value function approximation as a supervised learning problem. The (action-) value function of interest is assumed to be observed (either the value or action-value function of a given policy $\pi$, or directly the optimal Q-function), and it is projected onto the hypothesis space (minimizing $\left\|Q-\hat{Q}_{\theta}\right\|^{2}$ ) using a stochastic gradient descent or a recursive least-squares approach.

However, resulting algorithms make use of a value which is actually not observed. Bootstrapping consists in replacing this missing observation by a pseudo-observation computed by applying a sampled Bellman operator to the current estimate of the (action-) value function. This is an easy way to understand how to derive algorithms. However, bootstrapping is not supervised learning, the actual minimized cost function is provided in Sec. III-C.

\section{A. Bootstrapped Stochastic Gradient Descent}

Algorithms presented in this section aim at estimating respectively the value function of a given policy (TD), the Q-function of a given policy (TD-Q) or directly the optimal action-value function (Q-learning) by combining the bootstrapping principle with a stochastic gradient descent over the associated empirical cost function [2].

1) TD with Function Approximation: TD with function approximation (TD-VFA) aims at estimating the value function $V^{\pi}$ of a fixed policy $\pi$. Let the notation $v_{j}^{\pi}$ depict a (possibly noisy) observation of $V^{\pi}\left(s_{j}\right)$. The empirical cost is:

$$
\hat{J}_{V^{\pi}}(\theta)=\sum_{j}\left(v_{j}^{\pi}-\hat{V}_{\theta}\left(s_{j}\right)\right)^{2} .
$$

More precisely, TD with function approximation minimizes this empirical cost function using a stochastic gradient descent: parameters are adjusted by an amount proportional to an approximation of the gradient of (6), only evaluated on a single training example. Let $\alpha_{i}$ be a learning rate satisfying the classical stochastic approximation criterion: $\sum_{i=1}^{\infty} \alpha_{i}=\infty$ and $\sum_{i=1}^{\infty} \alpha_{i}^{2}<\infty$. Parameters are 


\begin{tabular}{|c|c|c|c|c|}
\cline { 2 - 5 } \multicolumn{1}{c|}{} & bootstrapping & residual & \multicolumn{2}{c|}{$\begin{array}{c}\text { projected fixed-point } \\
\text { direct / iterated }\end{array}$} \\
\hline $\begin{array}{c}\text { stochastic } \\
\text { gradient } \\
\text { descent }\end{array}$ & $\begin{array}{c}\text { TD-VFA [22] } \\
\text { TDQ-VFA [23] }\end{array}$ & R-SGD [9] & $\begin{array}{c}\text { (nl)GTD2 ([15])[14] } \\
\text { (nl)TDC ([15])[14] }\end{array}$ & \\
Greedy-GQ [17] & \\
\hline $\begin{array}{c}\text { (recursive) } \\
\text { least-squares }\end{array}$ & FPKF [8] & GPTD [25] & LSTD [12] & LSPE [18] \\
\hline slLSTD [13] & Q-OSP [19] \\
\hline other & & KTD [11] & fitted-Q [21] \\
\hline \multicolumn{4}{c}{ TABLE I } \\
SUMMARY.
\end{tabular}

updated according to the following Widrow-Hoff equation, given the $i^{\text {th }}$ observed state $s_{i}$ :

$$
\begin{aligned}
\theta_{i} & =\theta_{i-1}-\frac{\alpha_{i}}{2} \nabla_{\theta_{i-1}}\left(v_{i}^{\pi}-\hat{V}_{\theta}\left(s_{i}\right)\right)^{2} \\
& =\theta_{i-1}+\alpha_{i}\left(\nabla_{\theta_{i-1}} \hat{V}_{\theta}\left(s_{i}\right)\right)\left(v_{i}^{\pi}-\hat{V}_{\theta_{i-1}}\left(s_{i}\right)\right) .
\end{aligned}
$$

However, as mentioned above, the value of the state $s_{i}$ is not observed. It is where the bootstrapping principle applies. The unobserved value $v_{i}^{\pi}$ is replaced by an estimate computed by applying the sampled Bellman evaluation operator (4) to the current estimate $\hat{V}_{\theta_{i-1}}\left(s_{i}\right)$. Assume that not only the current state is observed, but the whole transition $\left(s_{i}, s_{i+1}\right)$ (sampled according to the policy $\pi$ ) as well as associated reward $r_{i}$. The corresponding update rule is therefore:

$$
\begin{gathered}
\theta_{i}=\theta_{i-1}+\alpha_{i}\left(\nabla_{\theta_{i-1}} \hat{V}_{\theta}\left(s_{i}\right)\right)\left(\hat{T}_{i}^{\pi} \hat{V}_{\theta_{i-1}}-\hat{V}_{\theta_{i-1}}\left(s_{i}\right)\right) \\
\text { with } \hat{T}_{i}^{\pi} \hat{V}_{\theta_{i-1}}=r_{i}+\gamma \hat{V}_{\theta_{i-1}}\left(s_{i+1}\right) .
\end{gathered}
$$

The idea behind using this sampled operator (and more generally behind bootstrapping) is twofold: if parameters are perfectly estimated and if the value function belongs to the hypothesis space, this provides an unbiased estimate of the actual value (the value function being the fixed-point of the unsampled operator) and this estimate provides more information as it is computed using the observed reward. Under some assumptions, notably a linear parameterization hypothesis, TD with function approximation can be shown to be convergent [26] (and it converges to the same solution as LSTD, described in Sec. V-A). This is no longer the case when it is combined with a nonlinear function approximator ${ }^{3}$ (a counterexample is exhibited in $[26])$.

2) TD-Q with Function Approximation: TD-Q with function approximation (TDQ-VFA) aims at estimating the Q-function of a fixed policy $\pi$. Combined with a control component such as an $\epsilon$-greedy or a Gibbs policy, it provides the well known SARSA algorithm (we use the name TD-Q to separate clearly policy evaluation from control, see Sec. VI). Notice that an MDP with a fixed policy defines a valued Markov chain over the state-action space, therefore value and Q-function evaluation are two similar problems. Let $q_{j}^{\pi}$ be a (possibly noisy) observation of $Q^{\pi}\left(s_{j}\right)$. The considered algorithm aims at minimizing

$$
\hat{J}_{Q^{\pi}}(\theta)=\sum_{j}\left(q_{j}^{\pi}-\hat{Q}_{\theta}\left(s_{j}, a_{j}\right)\right)^{2} .
$$

\footnotetext{
${ }^{3}$ Despite this, one of the important empirical successes of RL is based on TD with neural-network-based function approximation [27].
}

TD-Q with function approximation also minimizes the related empirical cost function using a stochastic gradient descent. Parameters are thus updated as follows, given the $i^{\text {th }}$ state-action pair $\left(s_{i}, a_{i}\right)$ :

$$
\begin{aligned}
\theta_{i} & =\theta_{i-1}-\frac{\alpha_{i}}{2} \nabla_{\theta_{i-1}}\left(q_{i}^{\pi}-\hat{Q}_{\theta}\left(s_{i}, a_{i}\right)\right)^{2} \\
& =\theta_{i-1}+\alpha_{i}\left(\nabla_{\theta_{i-1}} \hat{Q}_{\theta}\left(s_{i}, a_{i}\right)\right)\left(q_{i}^{\pi}-\hat{Q}_{\theta_{i-1}}\left(s_{i}, a_{i}\right)\right) .
\end{aligned}
$$

As before, $q_{i}^{\pi}$ is not observed and it is replaced by an estimate computed by applying the sampled Bellman evaluation operator to the current estimate $\hat{Q}_{\theta_{i-1}}\left(s_{i}, a_{i}\right)$. Assume that the whole transition $\left(s_{i}, a_{i}, s_{i+1}, a_{i+1}\right), a_{i+1}$ being sampled according to policy $\pi$, as well as associated reward $r_{i}$ are observed. Parameters are therefore updated according to:

$$
\begin{gathered}
\theta_{i}=\theta_{i-1}+\alpha_{i}\left(\nabla_{\theta_{i-1}} \hat{Q}_{\theta}\left(s_{i}, a_{i}\right)\right)\left(\hat{T}_{i}^{\pi} \hat{Q}_{\theta_{i-1}}-\hat{Q}_{\theta_{i-1}}\left(s_{i}, a_{i}\right)\right) \\
\text { with } \hat{T}_{i}^{\pi} \hat{Q}_{\theta_{i-1}}=r_{i}+\gamma \hat{Q}_{\theta_{i-1}}\left(s_{i+1}, a_{i+1}\right) .
\end{gathered}
$$

From a practical point of view, using the Q-function instead of the value function is of interest because it does not require the model to be known in order to derive a greedy policy. Convergence results holding for TD with function approximation apply rather directly to TD-Q with function approximation.

Introduced as above, TD-Q estimates the action-value function of the policy $\pi$ followed to sample the trajectory (3) used to feed the algorithm (on-policy learning). However, one may want to estimate the Q-function of some different target policy $\pi_{\mathrm{t}}$ (off-policy learning). This can be simply done by using the same transitions and replacing $\hat{T}_{i}^{\pi}$ by $\hat{T}_{i}^{\pi_{\mathrm{t}}}$. This means that the transition $\left(s_{i}, a_{i}, s_{i+1}, a_{i+1}\right)$ should be replaced by the transition $\left(s_{i}, a_{i}, s_{i+1}, a_{i+1}^{\mathrm{t}}\right)$, with $a_{i+1}^{\mathrm{t}}$ being sampled according to $\pi_{\mathrm{t}}\left(. \mid s_{t+1}\right)$. Also, one can consider an alternative bootstrap for $q_{\dot{t}}^{\pi_{\mathrm{t}}}$ (with $\pi=\pi_{\mathrm{t}}$ in the on-policy case). Instead of using $\hat{T}_{i}^{\pi_{\mathrm{t}}} \hat{Q}_{\theta_{i-1}}=r_{i}+\gamma \hat{Q}_{\theta_{i-1}}\left(s_{i+1}, a_{i+1}^{\mathrm{t}}\right)$, one can replace $q_{i}^{\pi_{\mathrm{t}}}$ by

$$
r_{i}+\gamma \mathbb{E}_{a \mid \pi_{\mathrm{t}}, s_{i+1}}\left[\hat{Q}_{\theta_{i-1}}\left(s_{i+1}, a\right)\right] .
$$

This is also an unbiased estimate of $\left[T^{\pi_{\mathrm{t}}} \hat{Q}_{\theta_{i-1}}\right]\left(s_{i}, a_{i}\right)$ which can be used whenever $\pi_{\mathrm{t}}$ is known and which may lead to better estimates [28]. These ideas (off-policy and alternative bootstrap) are quite general an can be applied to any policy evaluation algorithm presented in this survey (to any algorithm estimating a Q-function and derived from the Bellman evaluation operator). However, notice that if there is a too large mismatch between the 
stationary distributions induced by the policies $\pi$ and $\pi_{\mathrm{t}}$, the Q-function will be badly estimated by the offpolicy approach. For convergence properties of off-policy learning, see [29] and references therein.

3) Q-learning with Function Approximation: Q-learning with function approximation (QL-VFA) aims at estimating directly the optimal action-value function $Q^{*}$. Let $q_{j}^{*}$ be a (possibly noisy) observation of $Q^{*}\left(s_{j}\right)$. This algorithm aims at minimizing the empirical cost function:

$$
\hat{J}_{Q^{*}}(\theta)=\sum_{j}\left(q_{j}^{*}-\hat{Q}_{\theta}\left(s_{j}, a_{j}\right)\right)^{2} .
$$

The same approach is used, and parameters are recursively estimated using a stochastic gradient descent. Given the $i^{\text {th }}$ state-action pair $\left(s_{i}, a_{i}\right)$, parameters should be updated according to:

$$
\begin{aligned}
\theta_{i} & =\theta_{i-1}-\frac{\alpha_{i}}{2} \nabla_{\theta_{i-1}}\left(q_{i}^{*}-\hat{Q}_{\theta}\left(s_{i}, a_{i}\right)\right)^{2} \\
& =\theta_{i-1}+\alpha_{i}\left(\nabla_{\theta_{i-1}} \hat{Q}_{\theta}\left(s_{i}, a_{i}\right)\right)\left(q_{i}^{*}-\hat{Q}_{\theta_{i-1}}\left(s_{i}, a_{i}\right)\right) .
\end{aligned}
$$

As for preceding algorithms, the bootstrapping principle is applied to estimate the unobserved $q_{i}^{*}$ value, using the sampled Bellman optimality operator now, which assumes that the transition $\left(s_{i}, a_{i}, s_{i+1}\right)$ as well as associated reward $r_{i}$ are observed. Notice that Q-learning with function approximation is an off-policy algorithm, as it also evaluates a policy (the optimal one in this case) from samples generated according to a different policy. Practically, transitions can be sampled according to any sufficiently explorative policy. Parameters are updated as:

$$
\begin{gathered}
\theta_{i}=\theta_{i-1}+\alpha_{i}\left(\nabla_{\theta_{i-1}} \hat{Q}_{\theta}\left(s_{i}, a_{i}\right)\right)\left(\hat{T}_{i}^{*} \hat{Q}_{\theta_{i-1}}-\hat{Q}_{\theta_{i-1}}\left(s_{i}, a_{i}\right)\right) \\
\text { with } \hat{T}_{i}^{*} \hat{Q}_{\theta_{i-1}}=r_{i}+\gamma \max _{a \in A} \hat{Q}_{\theta_{i-1}}\left(s_{i+1}, a\right) .
\end{gathered}
$$

Under some assumptions, notably a linear parameterization and the fact that the sampling policy $\pi$ is explorative enough (it should visit all state-action pairs), QL-VFA can be shown to be convergent [30].

4) A Summary View: These algorithms can be formalized using the same unified notation. First, value and action-value function evaluation (TD and TD-Q with function approximation) are somehow redundant. As $V^{\pi}(s)=$ $\mathbb{E}_{a \mid \pi, s}\left[Q^{\pi}(s, a)\right]$, any algorithm aiming at estimating a Qfunction can easily be specialized to the related value function, as long as the policy is known (thus, this does not apply to Q-learning with function approximation). Practically, it consists in replacing the Q-function by the value function and state-action pairs by states. Consequently, value function estimation is not considered anymore in this article. Let $\hat{T}_{j}$ denote either the sampled evaluation or optimality operator, depending on the context. Let also $q_{j}$ be either $q_{j}^{\pi}$ or $q_{j}^{*}$, also depending on the context. TD$\mathrm{Q}$ and Q-learning with function approximation aim at minimizing the following empirical cost function, which is instantiated by specifying if evaluation or direct optimization is considered:

$$
\hat{J}(\theta)=\sum_{j}\left(q_{j}-\hat{Q}_{\theta}\left(s_{j}, a_{j}\right)\right)^{2} .
$$

As before, parameters are estimated using a stochastic gradient descent and by applying the bootstrapping principle, which leads to the following update:

$\theta_{i}=\theta_{i-1}+\alpha_{i}\left(\nabla_{\theta_{i-1}} \hat{Q}_{\theta}\left(s_{i}, a_{i}\right)\right)\left(\hat{T}_{i} \hat{Q}_{\theta_{i-1}}-\hat{Q}_{\theta_{i-1}}\left(s_{i}, a_{i}\right)\right)$.

A practical algorithm is instantiated by specifying which of the sampled Bellman operator is used for $\hat{T}$, that is $\hat{T}^{\pi}$ or $\hat{T}^{*}$. Algorithms have been detailed so far, for the sake of clarity. However, in the rest of this article this summarizing point of view is adopted. Notice also that this update is actually a Widrow-Hoff equation of the following form:

$$
\theta_{i}=\theta_{i-1}+K_{i} \delta_{i}
$$

In this expression, $\delta_{i}=\hat{T}_{i} \hat{Q}_{\theta_{i-1}}-\hat{Q}_{\theta_{i-1}}\left(s_{i}, a_{i}\right)$ is the so-called temporal difference (TD) error, which is the reward prediction error given the current estimate of the action-value function (it depends on which Bellman operator is considered) and $K_{i}=\alpha_{i} \nabla_{\theta_{i-1}} \hat{Q}_{\theta}\left(s_{i}, a_{i}\right)$ is a gain indicating in which direction the parameter vector should be corrected in order to improve the estimate. Most of (online) algorithms presented in this paper satisfy a Widrow-Hoff update.

\section{B. Bootstrapped Recursive Least-Squares}

The fixed-point Kalman Filter (FPKF) [8] also seeks at minimizing the empirical cost function linking (actually unobserved) action-values to the estimated Q-function (still with a bootstrapping approach):

$$
\hat{J}_{i}(\theta)=\sum_{j=1}^{i}\left(q_{j}-\hat{Q}_{\theta}\left(s_{j}, a_{j}\right)\right)^{2} .
$$

However, the parameterization is assumed to be linear and a (recursive) least-squares approach is adopted instead of the stochastic gradient descent used for preceding algorithms. The considered hypothesis space is of the form

$$
\mathcal{H}=\left\{\hat{Q}_{\theta}:(s, a) \in S \times A \rightarrow \phi(s, a)^{T} \theta \in \mathbb{R} \mid \theta \in \mathbb{R}^{p}\right\},
$$

where $\phi(s, a)$ is a feature vector (to be chosen beforehand). For a given state-action couple $\left(s_{j}, a_{j}\right), \phi\left(s_{j}, a_{j}\right)$ is shortened as $\phi_{j}$. The corresponding empirical objective function can thus be rewritten as:

$$
\hat{J}_{i}(\theta)=\sum_{j=1}^{i}\left(q_{j}-\phi_{j}^{T} \theta\right)^{2} .
$$

Thanks to linearity in parameters, this cost function is convex and has a unique minimum:

$$
\theta_{i}=\underset{\theta \in \mathbb{R}^{p}}{\operatorname{argmin}} \hat{J}_{i}(\theta) \text {. }
$$


This optimization problem can be solved analytically by zeroing the gradient of $\hat{J}_{i}(\theta)$; this is the principle of the least-squares method. Parameters are thus estimated as:

$$
\theta_{i}=\left(\sum_{j=1}^{i} \phi_{j} \phi_{j}^{T}\right)^{-1} \sum_{j=1}^{i} \phi_{j} q_{j} .
$$

Let us write $P_{i}^{-1}=\sum_{j=1}^{i} \phi_{j} \phi_{j}^{T}$. The Sherman-Morrison formula allows updating directly the inverse of a rank-one perturbed matrix:

$$
P_{i}=P_{i-1}-\frac{P_{i-1} \phi_{i} \phi_{i}^{T} P_{i-1}}{1+\phi_{i}^{T} P_{i-1} \phi_{i}} .
$$

This allows estimating parameters recursively:

$$
\theta_{i}=\theta_{i-1}+\frac{P_{i-1} \phi_{i}}{1+\phi_{i}^{T} P_{i-1} \phi_{i}}\left(q_{i}-\hat{Q}_{\theta_{i-1}}\left(s_{i}, a_{i}\right)\right) .
$$

As for algorithms presented in Sec. III-A, the bootstrapping principle is applied and the unobserved $q_{i}$ actionvalue is replaced by the estimate $\hat{T}_{i} \hat{Q}_{\theta_{i-1}}$ :

$$
\theta_{i}=\theta_{i-1}+\frac{P_{i-1} \phi_{i}}{1+\phi_{i}^{T} P_{i-1} \phi_{i}}\left(\hat{T}_{i} \hat{Q}_{\theta_{i-1}}-\hat{Q}_{\theta_{i-1}}\left(s_{i}, a_{i}\right)\right) .
$$

This equation is actually a Widrow-Hoff update (7). The temporal difference error is still $\delta_{i}=\hat{T}_{i} \hat{Q}_{\theta_{i-1}}-$ $\hat{Q}_{\theta_{i-1}}\left(s_{i}, a_{i}\right)$; this prediction error term is actually common to all algorithms aiming at estimating the action-value function. The gain depends on the fact that a least-squares minimization has been considered:

$$
K_{i}=\frac{P_{i-1} \phi_{i}}{1+\phi_{i}^{T} P_{i-1} \phi_{i}} .
$$

This gain can be compared to the one obtained using a stochastic gradient descent approach, presented in Sec. III-A. With a linear parametrization, the corresponding gain is $K_{i}=\alpha_{i} \phi_{i}$. Therefore, FPKF can be seen as a variation of TD-Q or Q-learning for which there is some automatic coordinate-based step-size adaptation.

If the sampled Bellman evaluation operator is considered, the FPKF update rule specializes as:

$$
\theta_{i}=\theta_{i-1}+K_{i}\left(r_{i}+\gamma \phi_{i+1}^{T} \theta_{i-1}-\phi_{i}^{T} \theta_{i-1}\right) .
$$

If the sampled Bellman optimality operator is considered, this update rule specializes as:

$\theta_{i}=\theta_{i-1}+K_{i}\left(r_{i}+\gamma \max _{a \in A}\left(\phi\left(s_{i+1}, a\right)^{T} \theta_{i-1}\right)-\phi_{i}^{T} \theta_{i-1}\right)$.

This algorithm can be shown to be convergent under some assumptions, for both sampled operators (evaluation and optimality in the case of optimal stopping problems). See [8] for details.

\section{Summary}

The principle of bootstrapping algorithms is to derive an online algorithm which minimizes a supervised-learningbased cost function:

$$
\theta_{i}=\underset{\omega \in \mathbb{R}^{p}}{\operatorname{argmin}} \sum_{j=1}^{i}\left(q_{j}-\hat{Q}_{\omega}\left(s_{j}, a_{j}\right)\right)^{2} .
$$

As values are not observed, the bootstrapping principle is applied: the unobserved $q_{j}$ value is replaced by some estimate. Given that we are looking for a fixed-point of one of the Bellman operators, this estimate is provided by $\hat{T}_{j} \hat{Q}_{\theta_{j-1}}$. Therefore, bootstrapping approaches solve the following optimization problem (as first noticed in [8]):

$$
\theta_{i}=\underset{\omega \in \mathbb{R}^{p}}{\operatorname{argmin}} \sum_{j=1}^{i}\left(\hat{T}_{j} \hat{Q}_{\theta_{j-1}}-\hat{Q}_{\omega}\left(s_{j}, a_{j}\right)\right)^{2} .
$$

This corresponds to Eq. (5) instantiated with $\xi=\theta_{j-1}$. Practical algorithms are then derived given that this cost function is minimized using a stochastic gradient descent or a recursive least-squares approach, and given what Bellman operator is considered. This is summarized in the first column of Tab. I on Page 5.

\section{Residual Approaches}

Residual approaches aim at finding an approximation of the fixed-point of one of the Bellman operators by minimizing the distance between the (action-) value function and its image through one of the Bellman operators. The associated cost function is:

$$
J(\theta)=\left\|\hat{Q}_{\theta}-T \hat{Q}_{\theta}\right\|^{2} .
$$

Practically, learning is done using samples and the Bellman operator is replaced by a sampled Bellman operator, the model (particularly transition probabilities) being unknown. The associated empirical cost function is therefore:

$$
\hat{J}(\theta)=\sum_{j}\left(\hat{Q}_{\theta}\left(s_{j}, a_{j}\right)-\hat{T}_{j} \hat{Q}_{\theta}\right)^{2} .
$$

A common drawback of all approaches aiming at minimizing this cost function is that they produce biased estimates of the (action-) value function. Basically, this is due to the fact that the expectation of a square is not the square of the expectation:

$$
\begin{aligned}
& \mathbb{E}_{s_{j+1} \mid s_{j}, a_{j}, \pi}\left[\left(Q\left(s_{j}, a_{j}\right)-\hat{T}_{j} Q\right)^{2}\right]= \\
& \left(Q\left(s_{j}, a_{j}\right)-(T Q)\left(s_{j}, a_{j}\right)\right)^{2}+\operatorname{Var}_{s_{j+1} \mid s_{j}, a_{j}, \pi}\left(\hat{T}_{j} Q\right) .
\end{aligned}
$$

There is an unwanted variance term acting as a penalty factor which favorises smooth functions. If such penalties are commonly used for regularization, this one is harmful here as it cannot be controlled [31]. All methods presented below can be modified so as to handle this problem, this will be shortly discussed. However, it is important to notice that any algorithm aiming at minimizing this cost presents this bias problem.

\section{A. Residual Stochastic Gradient Descent}

So-called residual algorithms (R-SGD for residual stochastic gradient descent) have been introduced in [9]. 
Their principle is to minimize the empirical cost function (8) using a stochastic gradient descent. The corresponding update rule is therefore:

$$
\begin{aligned}
& \theta_{i}=\theta_{i-1}-\frac{\alpha_{i}}{2} \nabla_{\theta_{i-1}}\left(\left(\hat{Q}_{\theta}\left(s_{i}, a_{i}\right)-\hat{T}_{i} \hat{Q}_{\theta}\right)^{2}\right) \\
& \text { with } \nabla_{\theta_{i-1}}\left(\left(\hat{Q}_{\theta}\left(s_{i}, a_{i}\right)-\hat{T}_{i} \hat{Q}_{\theta}\right)^{2}\right)= \\
& \left(\nabla_{\theta_{i-1}}\left(\hat{Q}_{\theta}\left(s_{i}, a_{i}\right)-\hat{T}_{i} \hat{Q}_{\theta}\right)\right)\left(\hat{T}_{i} \hat{Q}_{\theta_{i-1}}-\hat{Q}_{\theta_{i-1}}\left(s_{i}, a_{i}\right)\right) .
\end{aligned}
$$

Here again, this update is actually a Widrow-Hoff equation (see Eq. (7)) with $\delta_{i}=\hat{T}_{i} \hat{Q}_{\theta_{i-1}}-\hat{Q}_{\theta_{i-1}}\left(s_{i}, a_{i}\right)$ and $K_{i}=\alpha_{i} \nabla_{\theta_{i-1}}\left(\hat{Q}_{\theta}\left(s_{i}, a_{i}\right)-\hat{T}_{i} \hat{Q}_{\theta}\right)$. If the sampled Bellman evaluation operator is considered, gain and temporal difference error are given by:

$$
\begin{aligned}
K_{i} & =\alpha_{i} \nabla_{\theta_{i-1}}\left(\hat{Q}_{\theta}\left(s_{i}, a_{i}\right)-\gamma \hat{Q}_{\theta}\left(s_{i+1}, a_{i+1}\right)\right), \\
\delta_{i} & =r_{i}+\gamma \hat{Q}_{\theta_{i-1}}\left(s_{i+1}, a_{i+1}\right)-\hat{Q}_{\theta_{i-1}}\left(s_{i}, a_{i}\right) .
\end{aligned}
$$

A first problem arises when the sampled Bellman optimality operator is considered. In this case, we have:

$$
\begin{gathered}
K_{i}=\alpha_{i} \nabla_{\theta_{i-1}}\left(\hat{Q}_{\theta}\left(s_{i}, a_{i}\right)-\gamma \max _{a \in A} \hat{Q}_{\theta}\left(s_{i+1}, a\right)\right), \\
\delta_{i}=r_{i}+\gamma \max _{a \in A} \hat{Q}_{\theta_{i-1}}\left(s_{i+1}, a\right)-\hat{Q}_{\theta_{i-1}}\left(s_{i}, a_{i}\right) .
\end{gathered}
$$

Here, the gradient of the max operator must be computed: $\nabla_{\theta_{i-1}}\left(\max _{a \in A} \hat{Q}_{\theta}\left(s_{i+1}, a\right)\right)$. This is not straightforward; if this gain is introduced in [9], no solution is provided to compute it ${ }^{4}$. Another problem is that these algorithms compute biased estimates of the (action-) value function, as explained above. This is inherent to all approaches minimizing a residual cost function using a sampled Bellman operator. In order to handle this problem, it is proposed in [9] to use a double sampling scheme. Let us consider the Bellman evaluation operator. Two transitions are independently generated from the state-action couple $\left(s_{i}, a_{i}\right):\left(s_{i}, a_{i}, r_{i}^{\prime}, s_{i+1}^{\prime}, a_{i+1}^{\prime}\right)$ and $\left(s_{i}, a_{i}, r_{i}^{\prime \prime}, s_{i+1}^{\prime \prime}, a_{i+1}^{\prime \prime}\right)$. One of these transitions is used to compute the gain, and the other one to compute the TD error:

$$
\begin{aligned}
K_{i} & =\alpha_{i} \nabla_{\theta_{i-1}}\left(\hat{Q}_{\theta}\left(s_{i}, a_{i}\right)-\gamma \hat{Q}_{\theta}\left(s_{i+1}^{\prime}, a_{i+1}^{\prime}\right)\right), \\
\delta_{i} & =r_{i}+\gamma \hat{Q}_{\theta_{i-1}}\left(s_{i+1}^{\prime \prime}, a_{i+1}^{\prime \prime}\right)-\hat{Q}_{\theta_{i-1}}\left(s_{i}, a_{i}\right) .
\end{aligned}
$$

These two transitions being sampled independently, taking the expectation of $K_{i} \delta_{i}$ leads to the use of the true (that is unsampled) Bellman operator, without variance term contrary to the use of the same transition in both gain and TD error. However, this suggests that transitions can be sampled on demand (for example using a simulator), which might be a strong assumption.

\section{B. Residual Least-Squares}

In this section, methods based on a least-squares minimization of cost function (8) are reviewed. The parametric Gaussian Process Temporal Differences [10] algorithm

\footnotetext{
${ }^{4} \max _{a \in A} \hat{Q}_{\theta}\left(s_{i+1}, a\right)$ is generally non-differentiable resp. to $\theta$; a solution could be to rely on Fréchet subdifferentials [32].
}

minimizes it by assuming a linear parameterization as well as the Bellman evaluation operator, and the Kalman Temporal Differences framework [11] generalizes it to nonlinear parameterizations as well as to the Bellman optimality operator thanks to a statistical linearization approach [33].

1) Gaussian Process Temporal Differences: Assuming a linear parametrization and the Bellman evaluation operator, cost function (8) is linear and can be rewritten as:

$$
J_{i}(\theta)=\sum_{j=1}^{i}\left(r_{j}+\gamma \phi_{j+1}^{T} \theta-\phi_{j}^{T} \theta\right)^{2}
$$

Thanks to the linearity in parameters, it can be solved using a recursive least-squares approach: zeroing the gradient $\left(\nabla_{\theta} J_{i}(\theta)=0\right)$ provides a batch estimate which can be made recursive by using the Sherman-Morrison formula. To any least-squares problem can be linked a so called observation model, linking outputs to inputs through the hypothesized parametric model plus some white observation noise $n_{j}$. In this case, it is simply the sampled Bellman evaluation equation:

$$
r_{j}=\phi_{j}^{T} \theta-\gamma \phi_{j+1}^{T} \theta+n_{j} .
$$

With a unitary noise (that is $n_{j}$ is of unitary variance, $P_{n_{j}}=1$ ), the corresponding least-squares problem is provided by Eq. (9).

The (parametric) Gaussian Process Temporal Differences (GPTD) algorithm [10] considers the same observation model, with a not necessarily unitary noise $n_{j}$. The effect of the noise variance is to weight square terms in the minimized cost function, this is the slight difference with cost (9):

$$
J_{i}(\theta)=\sum_{j=1}^{i} \frac{1}{P_{n_{j}}}\left(r_{j}+\gamma \phi_{j+1}^{T} \theta-\phi_{j}^{T} \theta\right)^{2} .
$$

If one has some prior knowledge about the noise $n_{i}$, this can be used to obtain this simple weighted least-squares problem. If not, it is sufficient to consider a constant variance (therefore weighting no longer affects the solution of this optimization problem).

Let us write $\Delta \phi_{j}=\phi_{j}-\gamma \phi_{j+1}$. The unique parameter vector minimizing the above convex cost-function can be computed analytically by zeroing the gradient with respect to the parameter vector:

$\theta_{i}=\underset{\theta \in \mathbb{R}^{p}}{\operatorname{argmin}} J_{i}(\theta)=\left(\sum_{j=1}^{i} \frac{1}{P_{n_{j}}} \Delta \phi_{j} \Delta \phi_{j}^{T}\right)^{-1} \sum_{j=1}^{i} \frac{1}{P_{n_{j}}} \Delta \phi_{j} r_{j}$.

Let us write $P_{i}=\left(\sum_{j=1}^{i} \frac{1}{P_{n_{j}}} \Delta \phi_{j} \Delta \phi_{j}^{T}\right)^{-1}$. Thanks to the Sherman-Morrison formula, $P_{i}$ can be computed iteratively and parameters can be estimated recursively. Let $\theta_{0}$ and $P_{0}$ be some priors, the (parametric) GPTD algorithm is given by these two equations:

$$
\begin{aligned}
\theta_{i} & =\theta_{i-1}+\frac{P_{i-1} \Delta \phi_{i}}{P_{n_{i}}+\Delta \phi_{i}^{T} P_{i-1} \Delta \phi_{i}}\left(r_{i}-\Delta \phi_{i}^{T} \theta_{i-1}\right), \\
P_{i} & =P_{i-1}-\frac{P_{i-1} \Delta \phi_{i} \Delta \phi_{i}^{T} P_{i-1}}{P_{n_{i}}+\Delta \phi_{i}^{T} P_{i-1} \Delta \phi_{i}} .
\end{aligned}
$$


One can recognize the temporal difference error $\delta_{i}=$ $r_{i}-\Delta \phi_{i}^{T} \theta_{i-1}$ and a gain $K_{i}=\frac{P_{i-1} \Delta \phi_{i}}{P_{n_{i}}+\Delta \phi_{i}^{T} P_{i-1} \Delta \phi_{i}}$, to be linked again with the generic Widrow-Hoff update (7). Notice that $P_{i}$ is actually a variance matrix quantifying the uncertainty over current parameters estimation (it is the variance of the parameter vector conditioned on past $i$ observed rewards). This is not clear from the proposed leastsquares-based derivation, however it is direct by adopting a Bayesian perspective [25]. Notice that this interpretation of $P_{i}$ as being a variance matrix can be useful for handling the dilemma between exploration and exploitation [10].

As all other residual methods, GPTD produces biased estimates of the value function when transitions are stochastic. To alleviate this problem, a colored observation noise $n_{j}$ can be used instead of the classical white noise assumption (a noise being white if $\forall i \neq j, n_{i}$ and $n_{j}$ are independent, and a colored noise is any non-white noise) [34]. This noise allows removing the bias (because it leads to minimizing another cost function, linking state estimates to Monte Carlo samples of the discounted return [34][10, Ch. 4.4.3]), but it also induces a memory effect which prevents from learning in an off-policy manner, much like eligibility traces do (e.g., see [35]). These developments are not pursued here (they rely mainly on Bayesian inference and eligibility traces, just the later topic being briefly addressed in Sec. VIII).

The GPTD framework has been originally introduced in a different, non-parametric, manner [25]: the value function is modeled as a Gaussian process and a kernelbased online sparsification scheme is used to obtain a practical algorithm. As we focus on parametric value function approximation, the algorithm we review is the parametric GPTD [10, Sec. 4.3]. There also exists an LSTD-based variation of GPTD (see Sec. V-A for LSTD), derived from a maximum likelihood interpretation of the LSTD algorithm [10, Sec. 4.5]. In the parametric case, it is actually an $\ell_{2}$-regularized form of LSTD, so it is not further developed here.

2) Kalman Temporal Differences: As the GPTD framework, the Kalman Temporal Differences framework [11] also seeks at minimizing cost function (8), not necessarily considering a unitary noise variance too. Let us write $\hat{T}_{i} Q=r_{i}+\gamma \hat{P}_{i} Q$ with:

$\hat{P}_{i} Q=\left\{\begin{array}{l}Q\left(s_{i+1}, a_{i+1}\right) \text { (if sampled evaluation op.) } \\ \max _{a \in A} Q\left(s_{i+1}, a\right) \text { (if sampled optimality op.) }\end{array}\right.$

Estimated parameters should minimize:

$$
\hat{J}_{i}(\theta)=\sum_{j=1}^{i} \frac{1}{P_{n_{j}}}\left(r_{j}-\left(\hat{Q}_{\theta}\left(s_{j}, a_{j}\right)-\gamma \hat{P}_{j} \hat{Q}_{\theta}\right)\right)^{2} .
$$

Contrary to GPTD, KTD does not assume a linear parameterization nor the sampled evaluation operator. Instead, it makes use of a derivative-free linearization scheme (the derivative-free aspect allows considering the sampled optimality operator), the so-called statistical linearization [33]. The basic idea is to linearize this cost function (taking the distribution of parameters into account), which can subsequently be solved using a classical recursive leastsquares approach.

Linearization of (10) goes through the linearization of the associated observation model (to be considered as a function of $\theta$, the state-action couple being fixed):

$$
r_{j}=\hat{Q}_{\theta}\left(s_{j}, a_{j}\right)-\gamma \hat{P}_{j} \hat{Q}_{\theta}+n_{j}
$$

Assume that it is evaluated in $n$ sampled parameter vectors $\theta^{(k)}$ of associated weights $w_{k}$ (how to sample them practically and efficiently being addressed later):

$$
\left(\theta^{(k)}, r_{j}^{(k)}=\hat{Q}_{\theta^{(k)}}\left(s_{j}, a_{j}\right)-\gamma \hat{P}_{j} \hat{Q}_{\theta^{(k)}}\right)_{1 \leq k \leq n}
$$

The following statistics of interest are defined:

$$
\begin{aligned}
\bar{\theta} & =\sum_{k=1}^{n} w_{k} \theta^{(k)}, \quad \bar{r}_{j}=\sum_{k=1}^{n} w_{k} r_{j}^{(k)}, \\
P_{\theta} & =\sum_{k=1}^{n} w_{k}\left(\theta^{(k)}-\bar{\theta}\right)\left(\theta^{(k)}-\bar{\theta}\right)^{T}, \\
P_{\theta r_{j}} & =\sum_{k=1}^{n} w_{k}\left(\theta^{(k)}-\bar{\theta}\right)\left(r_{j}^{(k)}-\bar{r}_{j}\right)^{T}=P_{r_{j} \theta}^{T}, \\
P_{r_{j}} & =\sum_{k=1}^{n} w_{k}\left(r_{j}^{(k)}-\bar{r}_{j}\right)^{2} .
\end{aligned}
$$

Statistical linearization consists in linearizing the nonlinear observation model (11) around $\bar{\theta}$ (with $P_{\theta}$ being actually the spread of sampling) by adopting a statistical point of view. It finds a linear model $r_{j}=A_{j} \theta+b_{j}+u_{j}$, $u_{j}$ being a noise, by minimizing the sum of square errors between values of nonlinear and linearized functions in the regression points:

$$
\left(A_{j}, b_{j}\right)=\underset{A, b}{\operatorname{argmin}} \sum_{k=1}^{n}\left(e_{j}^{(k)}\right)^{2} \text { with } e_{j}^{(k)}=r_{j}^{(k)}-\left(A \theta^{(k)}+b\right)
$$

The solution of this optimization problem is given by:

$$
A_{j}=P_{r_{j} \theta} P_{\theta}^{-1} \text { and } b_{j}=\bar{r}_{j}-A_{j} \bar{\theta} \text {. }
$$

Moreover, it is easy to check that the covariance matrix of the error is given by:

$$
P_{e_{j}}=\sum_{k=1}^{n}\left(e_{j}^{(k)}\right)^{2}=P_{r_{j}}-A_{j} P_{\theta} A_{j}^{T}
$$

The nonlinear observation model (11) can thus be replaced by the following equivalent linear observation model:

$$
r_{j}=A_{j} \theta+b_{j}+u_{j} \text { with } u_{j}=e_{j}+n_{j} .
$$

Notice that the linearization error is taken into account through the noise $e_{j}$. Noises $e_{j}$ and $n_{j}$ being independent, the variance of $P_{u_{j}}$ is given by $P_{u_{j}}=P_{e_{j}}+P_{n_{j}}$. Given this statistically linearized observation model, the least- 
squares problem can be rewritten in a linear form:

$$
\begin{aligned}
\theta_{i} & =\underset{\theta \in \mathbb{R}^{p}}{\operatorname{argmin}}\left(\sum_{j=1}^{i} \frac{1}{P_{u_{j}}}\left(r_{j}-A_{j} \theta-b_{j}\right)^{2}\right) \\
& =\left(\sum_{j=1}^{i} \frac{1}{P_{u_{j}}} A_{j}^{T} A_{j}\right)^{-1} \sum_{j=1}^{i} A_{j}\left(r_{j}-b_{j}\right) .
\end{aligned}
$$

With this cost function, the higher is the statistical linearization error of a given transition (quantified by $P_{u_{j}}$ through $P_{e_{j}}$, the less the corresponding square term contributes to the cost. Using the Sherman-Morrison formula, a recursive update of this estimate can be obtained (expressing the gain $K_{i}$ directly as a function of the statistics of interest defining $A_{i}$ and $b_{i}$ and assuming some priors $\theta_{0}$ and $\left.P_{\theta_{0}}\right)$ :

$$
\begin{aligned}
K_{i} & =\frac{P_{\theta_{i} r_{i}}}{P_{u_{i}}+P_{r_{i}}}, \\
\theta_{i} & =\theta_{i-1}+K_{i}\left(r_{i}-\bar{r}_{i}\right), \\
P_{\theta_{i}} & =P_{\theta_{i-1}}-K_{i}\left(P_{v_{i}}+P_{r_{i}}\right) K_{i}^{T} .
\end{aligned}
$$

Here again, the gain's magnitude is directly linked to the linearization error, and large errors will result in small updates (this can be seen as an automatic learning rate). Eq. (14) corresponds also to a Widrow-Hoff update, with $r_{i}-\bar{r}_{i}$ being a statistically linearized temporal difference error $\left(\bar{r}_{i}\right.$ is a prediction of the reward given the currently estimated Q-function and the experienced transitions; the algorithm does not optimize a myopic TD error).

How to actually sample parameter vectors in order to perform statistical linearization is addressed now. With this recursive estimation, $\theta_{i-1}$ (the previous estimate) and $P_{i-1}$ (the associated uncertainty matrix, as explained in Sec. IV-B1) are known, and the issue is to compute $A_{i}$ and $b_{i}$. A first thing is to choose the point around which to linearize and with which magnitude. It is legitimate to sample around the previous estimate $\theta_{i-1}$ and with a spread related to the uncertainty of these estimates. In other words, $n$ parameter vectors are sampled such that $\bar{\theta}_{i}=\theta_{i-1}$ and $P_{\theta_{i}}=P_{i-1}$. Notice that $\bar{\theta}_{i}$, the point around which linearization is performed in order to update parameters, is different from $\theta_{i}$, the updated parameter vector. There remains the choice of how parameter vectors are sampled. A natural idea would be to assume a Gaussian distribution of mean $\theta_{i-1}$ and variance $P_{i-1}$ and to compute statistics of interest (Eqs. (12) to (13)) using a Monte Carlo approach. However, this would be particularly inefficient. Actually, the problem of sampling these points can be stated as follows: how to sample a random variable (here the parameter vector) of known mean and variance (here $\theta_{i-1}$ and $P_{i-1}$ ) in order to compute accurate estimates of first and second order moments of a nonlinear mapping of this random variable (here $\left.\hat{Q}_{\theta}\left(s_{i}, a_{i}\right)-\gamma \hat{P}_{i} \hat{Q}_{\theta}\right)$. The unscented transform [36] provides a solution to this problem and is used by the KTD framework ${ }^{5}$. Full details are provided in [11].

KTD generalizes GPTD in the sense that it allows handling nonlinearities: nonlinear parameterization thanks to the linearization, and the (sampled) Bellman optimality operator thanks to the fact that this linearization scheme does not rely on a gradient computation. Notice that KTD reduces to parametric GPTD if a linear parameterization as well as the sampled Bellman evaluation operator are considered. As other residual approaches, KTD suffers from the bias problem when system transitions are stochastic. In order to handle this issue, a colored noise model (based on the idea of eligibility traces) can be used [40] (which is actually a generalization of the noise proposed in [34] for GPTD). However, as mentioned in Sec. IV-B1, this induces some memory effects which prevent from learning in an off-policy manner. Consequently, the sampled Bellman optimality operator can no longer be considered in this setting, because of its off-policy aspect. Using a colored noise also leads to minimizing a different cost function. As for GPTD, these developments are not pursued here, see the corresponding papers [40][34][35]. Notice that the available uncertainty information (matrix $P_{i}$ ) can be useful for the dilemma between exploration and exploitation [41][42].

\section{Summary}

Residual approaches aim at finding an approximation of the fixed-point of one of the Bellman operators by minimizing the distance between the (action-) value function and its image through one of the Bellman operators. The associated theoretical cost function is $J(\theta)=\left\|\hat{Q}_{\theta}-T \hat{Q}_{\theta}\right\|^{2}$.

Practically, learning is done using samples and the Bellman operator is replaced by its sampled counterpart, the model being unknown. The associated empirical cost function is therefore:

$$
\theta_{i}=\underset{\omega \in \mathbb{R}^{p}}{\operatorname{argmin}} \sum_{j=1}^{i}\left(\hat{T}_{j} \hat{Q}_{\omega}-\hat{Q}_{\omega}\left(s_{j}, a_{j}\right)\right)^{2} .
$$

This is exactly Eq. (5) instantiated with $\xi=\omega$. Minimizing this cost function by considering one of the Bellman operator and using either a stochastic gradient descent or a recursive least-squares approach (possibly generalized using a statistical linearization) provides one of the presented algorithms (see the second column of Tab. I).

A common drawback of all approaches aiming at minimizing this cost function is that they produce biased estimates of the (action-) value function: minimizing the empirical cost function (8) does not lead to minimize the theoretical cost function $\left\|\hat{Q}_{\theta}-T \hat{Q}_{\theta}\right\|^{2}$ asymptotically. All methods presented in Sec. IV can be modified so as to handle the problem: a double sampling scheme is suggested in Sec. IV-A, and original methods based on coloring the

\footnotetext{
${ }^{5}$ Notice that other approximation schemes can be considered instead of the unscented transform, such as the scaled unscented transform [37], approximation schemes based on Sterling interpolation [38] or more generally sigma-point-based transforms [39].
} 
observation noise in Sections IV-B1 and IV-B2. Nevertheless, it is important to notice that any algorithm aiming at minimizing this cost presents this bias problem.

\section{Projected Fixed-Point Approaches}

Projected fixed-point approaches seek at minimizing the distance between the estimated action-value function and the projection (the projection operator being written $\Pi$ ) of the image of this function under a Bellman operator onto the hypothesis space $\mathcal{H}$ :

$$
J(\theta)=\left\|\hat{Q}_{\theta}-\Pi T \hat{Q}_{\theta}\right\|^{2} \text { with } \Pi f=\underset{\hat{f} \in \mathcal{H}}{\operatorname{argmin}}\|f-\hat{f}\|^{2} .
$$

This is illustrated in Fig. 1. The action-value function estimate $\hat{Q}_{\theta}$ lies in the hypothesis space $\mathcal{H}$. Its image under a Bellman operator $T \hat{Q}_{\theta}$ does not necessarily lye on this hypothesis space. Residual approaches of Sec. IV try to minimize the distance between these two functions, that is line (1) in Fig. 1, with the drawback that using a sampled Bellman operator leads to biased estimates, as discussed before. The function $T \hat{Q}_{\theta}$ can be projected onto the hypothesis space, this projection minimizing the distance between $T \hat{Q}_{\theta}$ and the hypothesis space (line (2) in Fig. 1). Projected fixed-point methods aim at minimizing the distance between this projection and $\hat{Q}_{\theta}$, represented by line (3) in Fig. 1.

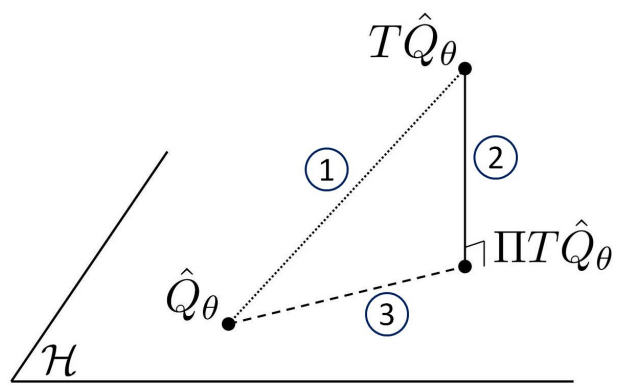

Fig. 1. Projected fixed-point principle.

Contrary to bootstrapping and residual approaches, least-squares-based algorithms have been introduced before stochastic-gradient-based ones for solving the projected fixed-point problem. Thus, they are reviewed first.

\section{A. Least-Squares-based Approaches}

This section reviews algorithms which use a leastsquares approach to minimize the empirical cost linked to $(15)$ :

$$
\begin{aligned}
\theta_{i} & =\underset{\theta \in \mathbb{R}^{p}}{\operatorname{argmin}} \sum_{j=1}^{i}\left(\hat{Q}_{\theta}\left(s_{j}, a_{j}\right)-\hat{Q}_{\omega_{\theta}}\left(s_{j}, a_{j}\right)\right)^{2} \\
\text { with } \omega_{\theta} & =\underset{\omega \in \mathbb{R}^{p}}{\operatorname{argmin}} \sum_{j=1}^{i}\left(\hat{Q}_{\omega}\left(s_{j}, a_{j}\right)-\hat{T}_{j} \hat{Q}_{\theta}\right)^{2} .
\end{aligned}
$$

Obviously, cost related to (16) is minimized for $\theta=\omega_{\theta}$ (assuming that this equation has a solution, which can at least be ensured for the evaluation operator and a linear parametrization). Therefore, nested optimization problems (16) and (17) can be summarized as $\theta_{i}=\omega_{\theta_{i}}$ :

$$
\theta_{i}=\underset{\omega \in \mathbb{R}^{p}}{\operatorname{argmin}} \sum_{j=1}^{i}\left(\hat{Q}_{\omega}\left(s_{j}, a_{j}\right)-\hat{T}_{j} \hat{Q}_{\theta_{i}}\right)^{2} .
$$

Notice that as $\theta_{i}$ appears in both sides of this equation, this is not a pure quadratic cost function, but a fixed-point problem. The least-squares temporal differences (LSTD) algorithm [12] assumes a linear parameterization and the (sampled) Bellman evaluation operator in order to solve the above optimization problem. The statistically linearized LSTD (slLSTD) algorithm [13] generalizes it to nonlinear parameterizations and to the (sampled) Bellman optimality operator thanks to a statistical linearization process (the generalization from LSTD to sILSTD being quite close to the generalization from GPTD to KTD).

1) Least-Squares Temporal Differences: $\operatorname{LSTD}^{6}$ assumes a linear parameterization as well as the sampled Bellman evaluation operator. Using the same notations as before, optimization problem (18) can be rewritten as:

$$
\theta_{i}=\underset{\omega \in \mathbb{R}^{p}}{\operatorname{argmin}} \sum_{j=1}^{i}\left(r_{j}+\gamma \phi_{j+1}^{T} \theta_{i}-\phi_{j}^{T} \omega\right)^{2} .
$$

Thanks to linearity in $\omega$ (linear parameterization assumption), this can be analytically solved:

$$
\theta_{i}=\left(\sum_{j=1}^{i} \phi_{j} \phi_{j}^{T}\right)^{-1} \sum_{j=1}^{i} \phi_{j}\left(r_{j}+\gamma \phi_{j+1}^{T} \theta_{i}\right) .
$$

Thanks to linearity in $\theta_{i}$ (linear parameterization and evaluation operator assumptions), the parameter vector can be isolated:

$$
\theta_{i}=\left(\sum_{j=1}^{i} \phi_{j}\left(\phi_{j}-\gamma \phi_{j+1}\right)^{T}\right)^{-1} \sum_{j=1}^{i} \phi_{j} r_{j} .
$$

Eq. (19) defines the (batch) LSTD estimate. Let us write $M_{i}^{-1}=\sum_{j=1}^{i} \phi_{j} \Delta \phi_{j}^{T}$. Thanks to the Sherman-Morrison formula, a recursive form of this estimation process can be obtained (assuming some priors $\theta_{0}$ and $M_{0}$ ):

$$
\begin{aligned}
K_{i} & =\frac{M_{i-1} \phi_{i}}{1+\left(\phi_{i}-\gamma \phi_{i+1}\right)^{T} M_{i-1} \phi_{i}}, \\
\theta_{i} & =\theta_{i-1}+K_{i}\left(r_{i}+\gamma \phi_{i+1}^{T} \theta_{i-1}-\phi_{i}^{T} \theta_{i-1}\right), \\
M_{i} & =M_{i-1}-K_{i}\left(M_{i-1}^{T}\left(\phi_{i}-\gamma \phi_{i+1}\right)\right)^{T} .
\end{aligned}
$$

Once again, $K_{i}$ is a gain and $r_{i}+\gamma \phi_{i+1}^{T} \theta_{i-1}-\phi_{i}^{T} \theta_{i-1}$ a temporal difference error, to be linked to the Widrow-Hoff update (7).

LSTD can be slightly modified to have an improved computational cost [45] (assuming that the features are sparse, which is not necessarily the case), and it can be "mixed" with a residual approach [46].

\footnotetext{
${ }^{6}$ Notice that LSTD has not been originally introduced as a projected fixed point method (like in [43]), but as a residual least-squares debiased using instrumental variables [44]. See [12] for details.
} 
2) Statistically Linearized LSTD: The sILSTD algorithm [13] generalizes LSTD: it does not assume a linear parameterization nor the Bellman evaluation operator. The corresponding optimization problem is therefore (this equation being valid thanks to the subsequent linearization):

$$
\theta_{i}=\underset{\omega \in \mathbb{R}^{p}}{\operatorname{argmin}} \sum_{j=1}^{i}\left(r_{j}+\gamma \hat{P}_{j} \hat{Q}_{\theta_{i}}-\hat{Q}_{\omega}\left(s_{j}, a_{j}\right)\right)^{2} .
$$

How sILSTD generalizes LSTD is very close to how KTD generalizes GPTD: a statistical linearization is performed, which allows solving this optimization problem analytically and recursively. Eq. (20) can be linked to the following observation model $\left(n_{j}\right.$ being here a unitary white and centered observation noise):

$$
r_{j}+\gamma \hat{P}_{j} \hat{Q}_{\theta_{i}}=\hat{Q}_{\omega}\left(s_{j}, a_{j}\right)+n_{j} .
$$

The noise is chosen unitary to strengthen parallel to LSTD, but extension to non-unitary noise is straightforward. As for KTD, a statistical linearization is performed. However, here two different quantities have to be linearized: $\hat{Q}_{\omega}\left(s_{j}, a_{j}\right)$ and $\hat{P}_{j} \hat{Q}_{\theta_{i}}$.

Assume that $n$ parameter vectors $\omega^{(k)}$ of associated weights $w_{k}$ are sampled (using the unscented transform), and that their images are computed:

$$
\left(\omega^{(k)}, q_{j}^{(k)}=\hat{Q}_{\omega^{(k)}}\left(s_{j}, a_{j}\right)\right)_{1 \leq k \leq n} .
$$

Using the statistical linearization process explained in Sec. IV-B2 and defining the statistics of interest accordingly to Eqs. (12) to (13), the following linear observation model is obtained:

$$
\begin{gathered}
\hat{Q}_{\omega}\left(s_{j}, a_{j}\right)=A_{j} \omega+b_{j}+e_{j} \\
\text { with } A_{j}=P_{q_{j} \omega} P_{\omega}^{-1}, b_{j}=\bar{q}_{j}-A_{j} \bar{\omega} \\
\text { and } P_{e_{j}}=P_{q_{j}}-A_{j} P_{\omega} A_{j}^{T} .
\end{gathered}
$$

Recall that the noise $e_{j}$ is centered and can be sampled as $e_{j}^{(k)}=q_{j}^{(k)}-\left(A_{j} \omega^{(k)}+b_{j}\right)$. The term $\hat{P} \hat{Q}_{\theta_{i}}\left(s_{j}, a_{j}\right)$ also needs to be linearized. Assume that $n$ parameter vectors $\theta_{i}^{(k)}$ of associated weights $w_{k}$ are sampled (again using the unscented transform), and that their images are computed:

$$
\left(\theta_{i}^{(k)}, p_{q_{j}}^{(k)}=\hat{P} \hat{Q}_{\theta_{i}^{(k)}}\left(s_{j}, a_{j}\right)\right)_{1 \leq k \leq n} .
$$

Using the statistical linearization process explained in Sec. IV-B2 and defining the statistics of interest accordingly to Eqs. (12) to (13), the following linear observation model is obtained:

$$
\begin{gathered}
\hat{P} \hat{Q}_{\theta_{i}}\left(s_{j}, a_{j}\right)=C_{j} \theta_{i}+d_{j}+\epsilon_{j} \\
\text { with } C_{j}=P_{p_{q_{j}} \theta_{i}} P_{\theta_{i}}^{-1}, d_{j}=\bar{p}_{q_{j}}-C_{j} \bar{\theta}_{i} \\
\text { and } P_{\epsilon_{j}}=P_{p_{q_{j}}}-C_{j} P_{\theta_{i}} C_{j}^{T} .
\end{gathered}
$$

Recall that the noise $\epsilon_{j}$ is centered and can be sampled as $\epsilon_{j}^{(k)}=p_{q_{j}}^{(k)}-\left(C_{j} \theta_{i}^{(k)}+d_{j}\right)$. Notice also that $\bar{\theta}_{i}$ is not equal to $\theta_{i}$ a priori.
Linearized models (22) and (23) can be injected into observation model (21):

$$
\begin{aligned}
r_{j}+\gamma\left(C_{j} \theta_{i}+d_{j}+\epsilon_{j}\right) & =A_{j} \omega+b_{j}+e_{j}+n_{j} \\
\Leftrightarrow r_{j}+\gamma\left(C_{j} \theta_{i}+d_{j}\right) & =A_{j} \omega+e_{j}-\gamma \epsilon_{j}+n_{j} .
\end{aligned}
$$

The linearization error is taken into account in the centered noise $u_{j}$ of variance $P_{u_{j}}$ :

$$
u_{j}=n_{j}+e_{j}-\gamma \epsilon_{j} \text { and } P_{u_{j}}=\mathbb{E}\left[u_{j}^{2}\right] .
$$

This equivalent observation model leads to the following optimization problem, which can be solved analytically:

$$
\begin{aligned}
\theta_{i} & =\underset{\omega \in \mathbb{R}^{p}}{\operatorname{argmin}} \sum_{j=1}^{i} \frac{1}{P_{u_{j}}}\left(r_{j}+\gamma\left(C_{j} \theta_{i}+d_{j}\right)-\left(A_{j} \omega+b_{j}\right)\right)^{2}(24) \\
& =\left(\sum_{j=1}^{i} \frac{1}{P_{u_{j}}} A_{j}^{T}\left(A_{j}-\gamma C_{j}\right)\right)^{-1} \sum_{j=1}^{i} \frac{1}{P_{u_{j}}} A_{j}\left(r_{j}+\gamma d_{j}-b_{j}\right) .
\end{aligned}
$$

This is the statistically linearized variation of optimization problem (20) (which explains the similarity between the LSTD batch estimate (19) and Eq. (25)). Also, similarly to what happens with KTD, the statistical linearization error is taken into account through the noise variance $P_{u_{j}}$ (the bigger the statistical linearization error, the lesser the impact of the related sample on the learning process). The Sherman-Morrison formula allows again deriving a recursive estimation of $\theta_{i}$. Assume that some priors $\theta_{0}$ and $M_{0}$ are chosen, the sILSTD algorithm is defined as:

$$
\begin{aligned}
K_{i} & =\frac{M_{i-1} A_{i}^{T}}{P_{u_{i}}+\left(A_{i}-\gamma C_{i}\right) M_{i-1} A_{i}^{T}}, \\
\theta_{i} & =\theta_{i-1}+K_{i}\left(r_{i}+\gamma d_{i}-b_{i}-\left(A_{i}-\gamma C_{i}\right) \theta_{i-1}\right), \\
M_{i} & =M_{i-1}-K_{i}\left(M_{i-1}^{T}\left(A_{i}-\gamma C_{i}\right)^{T}\right)^{T} .
\end{aligned}
$$

Notice that once again, this satisfies the Widrow-Hoff update, with a gain $K_{i}$ and a temporal difference error $r_{i}+\gamma d_{i}-b_{i}-\left(A_{i}-\gamma C_{i}\right) \theta_{i-1}$ (which can be shown to simplify as $r_{i}+\gamma \bar{p}_{q_{i}}-\bar{q}_{i}$, the TD error being more visible under this form). Given this recursive formulation, there still remains to choose how to sample parameter vectors (related to $\omega$ and $\theta_{i}$ ) in order to compute $A_{i}, b_{i}, C_{i}, d_{i}$ and $P_{u_{i}}$.

As for KTD, the unscented transform is used to sample these parameter vectors. The parameter vector $\omega$ to be considered is the solution of Eq. (24), that is the solution of the fixed-point problem $\theta_{i}=\omega_{\theta_{i}}$. In this recursive estimation context, it is legitimate to linearize around the last estimate $\theta_{i-1}$. The mean being chosen, the only remaining choice is the associated variance $P_{i-1}$. In [13], it is proposed to use the same variance matrix as would have been provided by a statistically linearized recursive leastsquares [47] used to perform supervised learning of the approximate action-value function given true observations of the Q-values. The fact that the unobserved actionvalues are not used to update the variance matrix tends 
to legitimate this choice. The associated matrix update is:

$$
P_{i}=P_{i-1}-\frac{P_{i-1} A_{i}^{T} A_{i} P_{i-1}}{1+A_{i} P_{i-1} A_{i}^{T}}
$$

The same approach is used to compute $C_{i}$ and $d_{i}$, coming from the statistical linearization of $\hat{P}_{i} \hat{Q}_{\theta_{i}}$. As before, the linearization is performed around the last estimate $\theta_{i-1}$ and considering the matrix variance $\Sigma_{i-1}$ provided by a statistical linearized recursive least-squares that would perform a supervised regression of $\hat{P}_{i} \hat{Q}_{\theta_{i}}$ :

$$
\Sigma_{i}=\Sigma_{i-1}-\frac{\Sigma_{i-1} C_{i}^{T} C_{i} \Sigma_{i-1}}{1+C_{i} \Sigma_{i-1} C_{i}^{T}}
$$

These choices being made, $A_{i}, b_{i}, C_{i}$ and $d_{i}$ can be computed, see [13] for details. A last thing is to compute the variance $P_{u_{i}}$ of the noise $u_{i}=n_{i}+e_{i}-\gamma \epsilon_{i}$. The noise $n_{i}$ is independent of others, and the variance of $e_{i}-\gamma \epsilon_{i}$ can be computed using the unscented transform, which provides an analytical expression for this variance.

Mixing all these elements, the sILSTD can be built. Notice that it can be easily shown that with a linear parameterization and the (sampled) Bellman evaluation operator, slLSTD indeed reduces to LSTD [13] (this relies on the fact that the unscented transform is no longer an approximation for a linear mapping). Notice also that sILSTD being a projected fixed-point approach, it does not suffers from the bias problem, even if the observation noise is assumed to be white (contrary to GPTD/KTD).

\section{B. Stochastic Gradient Descent-based Approaches}

Algorithms presented in this section aim at minimizing the same cost function, that is :

$$
\begin{gathered}
J_{i}(\theta)=\underset{\theta \in \mathbb{R}^{p}}{\operatorname{argmin}} \sum_{j=1}^{i}\left(\hat{Q}_{\theta}\left(s_{j}, a_{j}\right)-\hat{Q}_{\omega_{\theta}}\left(s_{j}, a_{j}\right)\right)^{2} \\
\text { with } \hat{Q}_{\omega_{\theta}}=\hat{\Pi} \hat{T} \hat{Q}_{\theta} .
\end{gathered}
$$

However, here a stochastic gradient descent approach is considered instead of the least-squares approach of the above section. Algorithms presented in Sec. V-B1, namely Gradient Temporal Difference 2 (GTD2) and Temporal Difference with Gradient Correction (TDC) [14], assume a linear parameterization and the (sampled) Bellman evaluation operator. Algorithms presented in Sec. V-B2, namely nonlinear GTD2 (nlGTD2) and nonlinear TD (nlTDC) [15], extend them to the case of a nonlinear parameterization. The (linear) TDC algorithm has also been extended to eligibility traces [16] and to the Bellman optimality operator [17], these extensions being briefly presented in Sec. V-B3.

1) Gradient Temporal Difference 2, Temporal Difference with Gradient Correction: GTD2 and TDC algorithms [14] aim at minimizing cost function (26) while considering the Bellman evaluation operator, and they differ on the route taken to express the gradient followed to perform the stochastic gradient descent. Both methods rely on a linear parameterization, and are based on a reworked expression of the cost function. Let $\hat{\mathbf{Q}}$ be:

$$
\hat{\mathbf{Q}}=\left(\begin{array}{lll}
\hat{Q}\left(s_{1}, a_{1}\right) & \ldots & \hat{Q}\left(s_{i}, a_{i}\right)
\end{array}\right)^{T} .
$$

Cost function (26) can be rewritten as:

$$
J_{i}(\theta)=\left\|\hat{\mathbf{Q}}_{\theta}-\hat{\mathbf{Q}}_{\omega_{\theta}}\right\|^{2}=\left(\hat{\mathbf{Q}}_{\theta}-\hat{\mathbf{Q}}_{\omega_{\theta}}\right)^{T}\left(\hat{\mathbf{Q}}_{\theta}-\hat{\mathbf{Q}}_{\omega_{\theta}}\right) .
$$

Let also $\Phi_{i}$ (respectively $\Phi_{i}^{\prime}$ ) be the $p \times i$ matrix which columns are the features $\phi\left(s_{j}, a_{j}\right)$ (respectively $\left.\phi\left(s_{j+1}, a_{j+1}\right)\right)$ :

$$
\begin{aligned}
\Phi_{i} & =\left[\begin{array}{lll}
\phi\left(s_{1}, a_{1}\right) & \ldots & \phi\left(s_{i}, a_{i}\right)
\end{array}\right] \\
\text { and } \Phi_{i}^{\prime} & =\left[\begin{array}{lll}
\phi\left(s_{2}, a_{2}\right) & \ldots & \phi\left(s_{i+1}, a_{i+1}\right)
\end{array}\right] .
\end{aligned}
$$

Let $R_{i}$ be the set of observed rewards:

$$
R_{i}=\left(\begin{array}{lll}
r_{1} & \ldots & r_{i}
\end{array}\right)^{T} .
$$

As the parameterization is linear and as the Bellman evaluation is considered, the Q-values and their images through the sampled operator are given as:

$$
\begin{aligned}
\hat{\mathbf{Q}}_{\theta} & =\Phi_{i}^{T} \theta, \\
\hat{T} \hat{\mathbf{Q}}_{\theta} & =R_{i}+\gamma\left(\Phi_{i}^{\prime}\right)^{T} \theta .
\end{aligned}
$$

$\hat{\mathbf{Q}}_{\omega_{\theta}}$ is the projection of $\hat{T} \hat{\mathbf{Q}}_{\theta}$ onto the hypothesis space. Let us write $\Pi_{i}$ the empirical projection:

$$
\hat{\mathbf{Q}}_{\omega_{\theta}}=\Phi_{i}^{T} \omega_{\theta}=\Pi_{i} \hat{T} \hat{\mathbf{Q}}_{\theta} \text { with } \Pi_{i}=\Phi_{i}^{T}\left(\Phi_{i} \Phi_{i}^{T}\right)^{-1} \Phi_{i} .
$$

Cost function (27) can thus be rewritten as:

$$
J_{i}(\theta)=\left\|\left(\Phi_{i}^{T} \theta-\Pi_{i}\left(R_{i}+\gamma\left(\Phi_{i}^{\prime}\right)^{T} \theta\right)\right)\right\|^{2} .
$$

Two basic properties of projection operator are useful here. First, $\Pi_{i} \Phi_{i}^{T} \theta=\Phi_{i}^{T} \theta$ (the hypothesis space is invariant under the projection operator). Second, $\Pi_{i} \Pi_{i}^{T}=\Pi_{i}$. Using these relationships, the cost can be rewritten as:

$$
J_{i}(\theta)=\left(\Phi_{i}^{T} \theta-R_{i}-\gamma\left(\Phi_{i}^{\prime}\right)^{T} \theta\right)^{T} \Pi_{i}\left(\Phi_{i}^{T} \theta-R_{i}-\gamma\left(\Phi_{i}^{\prime}\right)^{T} \theta\right) .
$$

Let $\delta_{j}(\theta)=r_{j}+\gamma \phi_{j+1}^{T} \theta-\phi_{j}^{T} \theta$ be the temporal difference error, $J_{i}(\theta)$ is finally given as:

$$
J_{i}(\theta)=\left(\sum_{j=1}^{i} \phi_{j} \delta_{j}(\theta)\right)^{T}\left(\sum_{j=1}^{i} \phi_{j} \phi_{j}^{T}\right)^{-1}\left(\sum_{j=1}^{i} \phi_{j} \delta_{j}(\theta)\right) .
$$

Notice that a Gradient Temporal Difference (GTD) algorithm has been first introduced by considering a slightly different cost function [48]:

$$
J_{i}^{\prime}(\theta)=\left(\sum_{j=1}^{i} \phi_{j} \delta_{j}(\theta)\right)^{T}\left(\sum_{j=1}^{i} \phi_{j} \delta_{j}(\theta)\right) .
$$

This explains why the algorithm of [14] is called GTD2.

The negative gradient of cost function (28) is given by:

$$
\begin{aligned}
& -\frac{1}{2} \nabla_{\theta} J_{i}(\theta)= \\
& \left(\sum_{j=1}^{i}\left(\phi_{j}-\gamma \phi_{j+1}\right) \phi_{j}^{T}\right)\left(\sum_{j=1}^{i} \phi_{j} \phi_{j}^{T}\right)^{-1}\left(\sum_{j=1}^{i} \delta_{j}(\theta) \phi_{j}\right) .
\end{aligned}
$$


In order to avoid a bias problem, a second modifiable parameter vector $\omega \in \mathbb{R}^{p}$ is used to form a quasi-stationary estimate of the term $\left(\sum_{j=1}^{i} \phi_{j} \phi_{j}^{T}\right)^{-1}\left(\sum_{j=1}^{i} \delta_{j}(\theta) \phi_{j}\right)$, this being called the weight-doubling trick. Parameter vector $\theta$ is updated according to a stochastic gradient descent:

$$
\theta_{i}=\theta_{i-1}+\alpha_{i}\left(\phi_{i}-\gamma \phi_{i+1}\right) \phi_{i}^{T} \omega_{i-1} .
$$

Their remains to find an update rule for $\omega_{i}$. In order to obtain a $O(p)$ algorithm, it is also estimated using a stochastic gradient descent [14]. One can remark that $\omega_{i}$ is actually the solution of a linear least-squares optimization problem:

$$
\begin{aligned}
\omega_{i} & =\left(\sum_{j=1}^{i} \phi_{j} \phi_{j}^{T}\right)^{-1} \sum_{j=1}^{i} \delta_{j}(\theta) \phi_{j} \\
& =\underset{\omega \in \mathbb{R}^{p}}{\operatorname{argmin}} \sum_{j=1}^{i}\left(\phi_{j}^{T} \omega-\delta_{j}(\theta)\right)^{2} .
\end{aligned}
$$

This suggests the following update rule for $\omega_{i}$ (minimization of Eq. (30) using a stochastic gradient descent):

$$
\omega_{i}=\omega_{i-1}+\beta_{i} \phi_{i}\left(\delta_{i}\left(\theta_{i-1}\right)-\phi_{i}^{T} \omega_{i-1}\right) .
$$

Learning rates satisfy the classical stochastic approximation criterion. Moreover, they are chosen such that $\beta_{i}=\eta \alpha_{i}$ with $\eta>0$. The GTD2 algorithm is thus:

$$
\begin{aligned}
\theta_{i} & =\theta_{i-1}+\alpha_{i}\left(\phi_{i}-\gamma \phi_{i+1}\right) \phi_{i}^{T} \omega_{i-1}, \\
\omega_{i} & =\omega_{i-1}+\beta_{i} \phi_{i}\left(\delta_{i}\left(\theta_{i-1}\right)-\phi_{i}^{T} \omega_{i-1}\right), \\
\text { with } \delta_{i}(\theta) & =r_{i}+\gamma \phi_{i+1}^{T} \theta-\phi_{i}^{T} \theta .
\end{aligned}
$$

Under some assumptions, this algorithm can be shown to be convergent to the fixed point of $\Pi T$ [14]. Notice that if this algorithm is derived from the gradient of an objective function, it is not a true stochastic gradient method, because the expected weight update direction may differ from the direction of the negative gradient of the objective function (it is a pseudo-gradient method) [4].

By expressing the gradient in a slightly different way, another algorithm called TDC can be derived. Rewrites Eq. (29):

$$
\begin{aligned}
& -\frac{1}{2} \nabla_{\theta} J_{i}(\theta)=\left(\sum_{j=1}^{i} \delta_{j}(\theta) \phi_{j}\right) \\
& \quad-\gamma\left(\sum_{j=1}^{i} \phi_{j+1} \phi_{j}^{T}\right)\left(\sum_{j=1}^{i} \phi_{j} \phi_{j}^{T}\right)^{-1}\left(\sum_{j=1}^{i} \delta_{j}(\theta) \phi_{j}\right) .
\end{aligned}
$$

This gives rise to the following update for $\theta, \omega$ being updated as before:

$$
\theta_{i}=\theta_{i-1}+\alpha_{i} \phi_{i} \delta_{i}\left(\theta_{i-1}\right)-\alpha_{i} \gamma \phi_{i+1} \phi_{i}^{T} \omega_{i-1} .
$$

This algorithm is called TD with gradient correction because the first term, $\alpha_{i} \phi_{i} \delta_{i}\left(\theta_{i-1}\right)$, is the same as for TD with function approximation (see Sec. III-A) under a linear function approximation architecture, and the second term, $-\alpha_{i} \gamma \phi_{i+1} \phi_{i}^{T} \omega_{i-1}$, acts as a correction. For TDC, learning rates $\alpha_{i}$ and $\beta_{i}$ are chosen such as satisfying the classic stochastic approximation criterion, and such that $\lim _{i \rightarrow \infty} \frac{\alpha_{i}}{\beta_{i}}=0$. This means that $\theta_{i}$ is updated on a slower time-scale. The idea behind this is that $\omega_{i}$ should look stationary from the $\theta_{i}$ point of view. The TDC algorithm can be summarized as follows:

$$
\begin{aligned}
\theta_{i} & =\theta_{i-1}+\alpha_{i} \phi_{i} \delta_{i}\left(\theta_{i-1}\right)-\alpha_{i} \gamma \phi_{i+1} \phi_{i}^{T} \omega_{i-1}, \\
\omega_{i} & =\omega_{i-1}+\beta_{i} \phi_{i}\left(\delta_{i}\left(\theta_{i-1}\right)-\phi_{i}^{T} \omega_{i-1}\right),
\end{aligned}
$$

with $\delta_{i}(\theta)=r_{i}+\gamma \phi_{i+1}^{T} \theta-\phi_{i}^{T} \theta$.

This algorithm can also be shown to be convergent (also to $\Pi T$ ) under some assumptions [14].

2) Nonlinear extensions: in [15], GTD2 and TDC are extended to the case of a general nonlinear parameterization $\hat{Q}_{\theta}$, as long as it is differentiable with respect to $\theta$. The corresponding hypothesis space $\mathcal{H}=\left\{\hat{Q}_{\theta} \mid \theta \in \mathbb{R}^{p}\right\}$ is a differentiable submanifold onto which projecting is generally not computationally feasible. One may assume that the parameter vector $\theta$ is slightly updated in one step (given that learning rate are usually small), which causes the surface of the submanifold to be close to linear. Therefore, projection is done onto the tangent plane defined as $\mathcal{T} \mathcal{H}=\left\{(s, a) \in S \times A \rightarrow \omega^{T} \nabla_{\theta} \hat{Q}_{\theta}(s, a) \mid \omega \in \mathbb{R}^{p}\right\}$ (in other words, linearization of $\hat{Q}_{\theta}(s, a)$ by a first order Taylor expansion). The corresponding projection operator $\Pi_{i}^{\theta}$ can be obtained as in Sec. V-B1, the tangent space being an hyperplane:

$$
\begin{aligned}
\Pi_{i}^{\theta} & =\left(\Phi_{i}^{\theta}\right)^{T}\left(\Phi_{i}^{\theta}\left(\Phi_{i}^{\theta}\right)^{T}\right)^{-1} \Phi_{i}^{\theta}, \\
\text { with } \Phi_{i}^{\theta} & =\left[\begin{array}{lll}
\nabla_{\theta} \hat{Q}_{\theta}\left(s_{1}, a_{1}\right) & \ldots & \nabla_{\theta} \hat{Q}_{\theta}\left(s_{i}, a_{i}\right) .
\end{array}\right]
\end{aligned}
$$

The corresponding cost function can therefore be derived as in Sec. V-B1, with basically feature vectors $\phi(s, a)$ being replaced by linearized features $\phi_{j}^{\theta}=\nabla_{\theta} \hat{Q}_{\theta}\left(s_{j}, a_{j}\right)$, and by writing $\delta_{j}(\theta)$ the temporal difference error $r_{j}+$ $\gamma \hat{Q}_{\theta}\left(s_{j+1}, a_{j+1}\right)-\hat{Q}_{\theta}\left(s_{j}, a_{j}\right)$ :

$$
J_{i}(\theta)=\left(\sum_{j=1}^{i} \phi_{j}^{\theta} \delta_{j}(\theta)\right)^{T}\left(\sum_{j=1}^{i} \phi_{j}^{\theta}\left(\phi_{j}^{\theta}\right)^{T}\right)^{-1}\left(\sum_{j=1}^{i} \phi_{j}^{\theta} \delta_{j}(\theta)\right) .
$$

The gradient of this cost function is [15]:

$$
\begin{aligned}
& -\frac{1}{2} \nabla_{\theta} J_{i}(\theta) \\
& =\left(\sum_{j=1}^{i}\left(\phi_{j}^{\theta}-\gamma \phi_{j+1}^{\theta}\right)\left(\phi_{j}^{\theta}\right)^{T}\right) \omega_{i}+h\left(\theta, \omega_{i}\right) \\
& =\sum_{j=1}^{i} \delta_{j}(\theta) \phi_{j}^{\theta}-\gamma\left(\sum_{j=1}^{i} \phi_{j+1}^{\theta}\left(\phi_{j}^{\theta}\right)^{T}\right) \omega_{i}+h\left(\theta, \omega_{i}\right) \\
& \text { with } \omega_{i}=\left(\sum_{j=1}^{i} \phi_{j}^{\theta}\left(\phi_{j}^{\theta}\right)^{T}\right)^{-1}\left(\sum_{j=1}^{i} \delta_{j}(\theta) \phi_{j}^{\theta}\right) \\
& \text { and } h(\theta, \omega)=-\sum_{j=1}^{i}\left(\delta_{j}(\theta)-\left(\phi_{j}^{\theta}\right)^{T} \omega\right)\left(\nabla^{2} \hat{Q}_{\theta}\left(s_{j}, a_{j}\right)\right) \omega .
\end{aligned}
$$


GTD2 and TDC are generalized to nlGTD2 and nlTDC using a stochastic gradient descent on the above cost function. Parameter vector $\omega_{i}$ is updated as in Sec. V-B1:

$$
\omega_{i}=\omega_{i-1}+\beta_{i} \phi_{i}^{\theta_{i-1}}\left(\delta_{i}\left(\theta_{i-1}\right)-\left(\phi_{i}^{\theta_{i-1}}\right)^{T} \omega_{i-1}\right) .
$$

The nonlinear GTD2 algorithm performs a stochastic gradient descent according to Eq. (31):

$$
\theta_{i}=\theta_{i-1}+\alpha_{i}\left(\left(\phi_{i}^{\theta_{i-1}}-\gamma \phi_{i+1}^{\theta_{i-1}}\right)\left(\phi_{i}^{\theta_{i-1}}\right)^{T} \omega_{i-1}-h_{i}\right)
$$

with

$$
h_{i}=\left(\delta_{i}\left(\theta_{i-1}\right)-\left(\phi_{i}^{\theta_{i-1}}\right)^{T} \omega_{i-1}\right)\left(\nabla_{\theta_{i-1}}^{2} \hat{Q}_{\theta}\left(s_{i}, a_{i}\right)\right) \omega_{i-1} .
$$

Learning rates are chosen as for the TDC algorithm, that is satisfying the classic stochastic approximation criterion and such that $\lim _{i \rightarrow \infty} \frac{\alpha_{i}}{\beta_{i}}=0$, which means that $\theta$ is updated on a slower timescale than $\omega$. The nonlinear TDC algorithm performs a stochastic gradient descent according to Eq. (32):

$\theta_{i}=\theta_{i-1}+\alpha_{i}\left(\phi_{i}^{\theta_{i-1}} \delta_{i}\left(\theta_{i-1}\right)-\gamma \phi_{i+1}^{\theta_{i-1}}\left(\phi_{i}^{\theta_{i-1}}\right)^{T} \omega_{i-1}-h_{i}\right)$

with

$h_{i}=\left(\delta_{i}\left(\theta_{i-1}\right)-\left(\phi_{i}^{\theta_{i-1}}\right)^{T} \omega_{i-1}\right)\left(\nabla_{\theta_{i-1}}^{2} \hat{Q}_{\theta}\left(s_{i}, a_{i}\right)\right) \omega_{i-1}$.

Learning rates are chosen as above. Both nlGTD2 and nITDC can be shown to be convergent to an undefined local minimum under some assumptions [15].

3) Extensions of TDC: the TDC algorithm (see Sec. V-B1) has been extended to eligibility traces in [16]. Moreover, this algorithm, called $\operatorname{GQ}(\lambda)$, allows off-policy learning, that is learning the value of one target policy while following another behaviorial policy. This new algorithm (for which some convergence guarantees are provided) still minimizes the empirical cost function linked to (15). However, instead of the $T^{\pi}$ Bellman operator considered so far, an eligibility-based $T_{\lambda}^{\pi}$ operator is used ( $\lambda$ being the eligibility factor), this operator being defined as the expected $\lambda$-return. See [16] for more details. Using eligibility traces induce a memory effect which prevents from learning in an off-policy manner without caution, see [35] for example. To cope with this problem, importance sampling [49] is used in [16] (the idea of using importance sampling for value function approximation having been first introduced in [35]). They present GQ $(\lambda)$ as an extension of Q-learning, which can be misleading. Actually, they consider off-policy learning (with a known and fixed target policy), but not the Bellman optimality operator.

Nevertheless, the TDC algorithm has also been extended to this operator [17] (this new algorithm being called Greedy-GQ). To do so, they consider the Bellman evaluation operator $T^{\pi_{\theta}}$ for a policy $\pi_{\theta}$ which depends on the currently estimated action-value function (through parameters $\theta$ ). Therefore, the considered policy is nonstationary (it evolves with parameters' estimation). If $\pi_{\theta}$ is greedy with respect to the learnt value function, then it is equivalent to considering the Bellman optimality operator. However, in this case, there are some non-differentiability problems (due to the max operator), and Fréchet subdifferentials are used to provide the algorithm [17]. A convergence analysis for these algorithms is also provided [17].

\section{Iterative projected fixed point}

Methods presented so far in Sec. V aim at minimizing the distance between the action-value function and the projection of the image of this function under a Bellman operator onto the hypothesis space:

$$
J(\theta)=\left\|\hat{Q}_{\theta}-\Pi T \hat{Q}_{\theta}\right\|^{2} .
$$

This can be interpreted as trying to find a fixed-point of the operator $\Pi T$, which is the composition of the projection operator $\Pi$ and of one of the Bellman operator $T$. Assuming that this operator is a contraction (which is not always the case, it depends on the projection operator), there exists a unique fixed-point which can be found by iterating the application of the $\Pi T$ operator:

$$
\hat{Q}_{\theta_{i}}=\Pi T \hat{Q}_{\theta_{i-1}} .
$$

Methods presented in this section adopt this point of view to provide algorithms.

1) Fitted-Q: Fitted-Q is a batch algorithm. It assumes that a set of $N$ transitions is available beforehand:

$$
\left\{\left(s_{j}, a_{j}, r_{j}, s_{j+1}, a_{j+1}\right)\right\}_{1 \leq j \leq N} .
$$

An initial Q-function $\hat{Q}_{\theta_{0}}$ is considered, and estimates are refined by iterating the (sampled) $\Pi T$ operator:

$$
\hat{Q}_{\theta_{i}}=\hat{\Pi} \hat{T} \hat{Q}_{\theta_{i-1}}, \quad \forall i>0 .
$$

Some important comments have to be made here. A sampled Bellman operator is used, because as usual transition probabilities are unknown. Most of time, fitted-Q suggests the sampled Bellman optimality operator, but this approach is of course still valid for the sampled Bellman evaluation operator. The $\Pi$ operator indeed represents any supervised learning algorithm (which can be more or less directly seen as a projection). Notice that the representation of the estimated action-value function is not necessarily parametric (e.g., using a tree-based supervised learning algorithm [21]).

A practical example is given now. Assume that at iteration $i$ the estimate $\hat{Q}_{\theta_{i-1}}$ is available. A first thing is to compute the image of this estimate through the sampled Bellman (here optimality) operator. This consists in computing the following training base, composed of state-action couples and estimated optimal Qvalues: $\left\{\left(s_{j}, a_{j}, r_{j}+\gamma \max _{a \in A} \hat{Q}_{\theta_{i-1}}\left(s_{j+1}, a\right)\right)\right\}_{1 \leq j \leq N}$. A supervised learning algorithm is then used on this set, associating inputs $\left(s_{j}, a_{j}\right)$ to estimated outputs $r_{j}+$ $\gamma \max _{a \in A} \hat{Q}_{\theta_{i-1}}\left(s_{j+1}, a\right)$. This iteration is repeated until a stopping criterion is met (e.g., a maximum number of steps or little changes in the representation). 
The fitted-Q idea probably dates back to [50]. Its convergence properties have been analyzed in [20], who reasons on the contraction property of the $\Pi T$ operator. In [51], its performance bounds in $\ell_{p}$ norm is analyzed. This is particularly judicious: if performance bounds of supervised learning algorithms are very often analyzed in $\ell_{p}$ norm, this is not the case for (approximate) dynamic programming which is most of the time analyzed in $\ell_{\infty}$ norm. Fitted-Q has been considered with many different function approximators. For example, see [52] for fitted-Q with a kernel-based regression, [53] for fitted-Q with neural networks trained by backpropagation or [21] for fitted-Q with tree-based methods.

2) Least-Squares Policy Evaluation: The least-squares policy evaluation (LSPE) algorithm has been proposed ${ }^{7}$ in [18]. It is directly introduced using the concept of eligibility traces, but this aspect is left apart in this article. The LSPE algorithm can be roughly seen as a fitted-Q algorithm using a linear parameterization, the (sampled) Bellman evaluation operator and for which a new training sample is added to the training set at each iteration. Thanks to linearity (linear parameterization and evaluation operator), an efficient online algorithm can be obtained.

The LSPE algorithm solves recursively $\hat{Q}_{\theta_{i}}=\Pi_{i} \hat{T} \hat{Q}_{\theta_{i-1}}$, the $\Pi_{i}$ projection operator being defined in Sec. V-B1. Given linearity, this can be rewritten as:

$$
\begin{aligned}
\theta_{i} & =\underset{\theta \in \mathbb{R}^{p}}{\operatorname{argmin}} \sum_{j=1}^{i}\left(\phi_{j}^{T} \theta-r_{j}-\gamma \phi_{j+1}^{T} \theta_{i-1}\right)^{2} \\
& =\left(\sum_{j=1}^{i} \phi_{j} \phi_{j}^{T}\right)^{-1} \sum_{j=1}^{i} \phi_{j}\left(r_{j}+\gamma \phi_{j+1}^{T} \theta_{i-1}\right) \\
& =\theta_{i-1}+\left(\sum_{j=1}^{i} \phi_{j} \phi_{j}^{T}\right)^{-1} \sum_{j=1}^{i} \phi_{j}\left(r_{j}-\left(\phi_{j}-\gamma \phi_{j+1}\right)^{T} \theta_{i-1}\right) .
\end{aligned}
$$

All terms involved can be computed recursively and efficiently, using notably the Sherman-Morrison formula:

$$
\begin{aligned}
B_{i}^{-1} & =\left(\sum_{j=1}^{i} \phi_{j} \phi_{j}^{T}\right)^{-1}=B_{i-1}^{-1}-\frac{B_{i-1}^{-1} \phi_{i} \phi_{i}^{T} B_{i-1}^{-1}}{1+\phi_{i}^{T} B_{i-1}^{-1} \phi_{i}}, \\
A_{i} & =\sum_{j=1}^{i} \phi_{j}\left(\phi_{j}-\gamma \phi_{j+1}\right)^{T}=A_{i-1}+\phi_{i}\left(\phi_{i}-\gamma \phi_{i+1}\right)^{T}, \\
b_{i} & =\sum_{j=1}^{i} \phi_{j} r_{j}=b_{i-1}+\phi_{i} r_{i} .
\end{aligned}
$$

The LSPE update can therefore be expressed in a form close to a Widrow-Hoff update:

$$
\theta_{i}=\theta_{i-1}+B_{i}^{-1}\left(b_{i}-A_{i} \theta_{i-1}\right) .
$$

${ }^{7}$ Actually, if the name LSPE has been introduced in [18] (in a multistep value-iteration context), the related algorithm has first been introduced in [54], where it is built upon $\lambda$-policy-iteration.
Actually, the way LSPE is presented here differs from [18] in the sense that the $B_{i}^{-1}$ matrix is originally scaled with a learning rate. The algorithm presented here is the case where the learning rate is chosen constant and equal to one. This algorithm (with a constant learning rate equal to one) is shown to be convergent in [55]. Notice that ideas behind LSPE have other applications [56] and can also be linked to variational inequalities [57].

3) Q-learning for optimal stopping problems: The Qlearning for optimal stopping problems (Q-OSP) [19] extends LSPE to the (sampled) Bellman optimality operator, the parameterization being still linear. This algorithm is originally presented in the case of optimal stopping problems, which are a restrictive class of Markovian decision processes. However, it is presented here in the general case.

The derivation of this algorithm is the same as for the LSPE one, by considering the optimality operator instead of the evaluation one:

$$
\begin{aligned}
\theta_{i} & =\underset{\theta \in \mathbb{R}^{p}}{\operatorname{argmin}} \sum_{j=1}^{i}\left(\phi_{j}^{T} \theta-r_{j}-\gamma \max _{a \in A}\left(\phi\left(s_{j+1}, a\right)^{T} \theta_{i-1}\right)\right) \\
& =\left(\sum_{j=1}^{i} \phi_{j} \phi_{j}^{T}\right)^{-1} \sum_{j=1}^{i}\left(r_{j}+\gamma \max _{a \in A}\left(\phi\left(s_{j+1}, a\right)^{T} \theta_{i-1}\right)\right) .
\end{aligned}
$$

The matrix $\left(\sum_{j=1}^{i} \phi_{j} \phi_{j}^{T}\right)^{-1}$ can still be computed recursively and efficiently. However, this is not the case for the term $\sum_{j=1}^{i}\left(r_{j}+\gamma \max _{a \in A}\left(\phi\left(s_{j+1}, a\right)^{T} \theta_{i-1}\right)\right)$ which requires remembering the whole trajectory and needs to be computed at each iteration. In [19] this algorithm is shown to be convergent for optimal stopping problems and under some assumptions, and some more computationally efficient variations for the same restrictive class of MDPs are also proposed.

\section{Summary}

Projected fixed-point approaches seek at estimating a fixed-point of the composed operator $\Pi T, \Pi$ being the projection onto the hypothesis space $\mathcal{H}$ and $T$ being one of the Bellman operator. This fixed-point can be computed directly, which corresponds to minimizing $\left\|\hat{Q}_{\theta}-\Pi T \hat{Q}_{\theta}\right\|^{2}$. The associated optimization problem is given by:

$$
\theta_{i}=\underset{\omega \in \mathbb{R}^{p}}{\operatorname{argmin}} \sum_{j=1}^{i}\left(\hat{Q}_{\omega}\left(s_{j}, a_{j}\right)-\hat{T}_{j} \hat{Q}_{\theta_{i}}\right)^{2} .
$$

This is Eq. (5) instantiated with $\xi=\theta_{i}$. If solved with a recursive least-squares approach, it gives the LSTD algorithm (or its statistical-linearization-based generalization). If solved using a stochastic gradient descent, it can provide GTD2 and TDC as well as their generalization to nonlinear parametrization, and their extensions to offpolicy learning and to the Bellman optimality operator $(\mathrm{GQ}(\lambda)$ and greedy GQ).

Other approaches search for this fixed point using an iterative method: $\hat{Q}_{\theta_{i}}=\Pi T \hat{Q}_{\theta_{i-1}}$. The corresponding 
optimization problem is:

$$
\theta_{i}=\underset{\omega \in \mathbb{R}^{p}}{\operatorname{argmin}} \sum_{j=1}^{i}\left(\hat{Q}_{\omega}\left(s_{j}, a_{j}\right)-\hat{T}_{j} \hat{Q}_{\theta_{i-1}}\right)^{2} .
$$

This is Eq. (5) instantiated with $\xi=\theta_{i-1}$. Solved using a least-squares approach, it provides LSPE or Q-OSP, given what Bellman operator is considered. In a batch setting and using any supervised learning algorithm as the projection, it is fitted-Q. See the third column of Tab. I.

\section{Control}

The ultimate goal of reinforcement learning is the control problem, that is computing an optimal control policy. We show here how the studied value function approximators integrate in the more general control problem.

\section{A. Batch learning}

Assume that a set of trajectories, sampled according to some (explorative enough) behaviorial policy, is available. In a batch setting, the aim is to estimate an optimal policy from the available data. To do so, approximate dynamic programming (ADP) can be used.

Approximate policy iteration (API) is generally speaking a policy iteration scheme where the policy evaluation step is approximated. API is initialized with some policy $\pi_{0}$. At each time step $k$, policy $\pi_{k}$ is evaluated using any approximate policy evaluation algorithm fed with the available data (that is, any action-value function approximator based on the Bellman evaluation operator and used in an off-policy setting, as explained in Sec. III-A2). This gives an estimate $\hat{Q}_{\theta}^{\pi_{k}}$, which is used to improve the policy: $\pi_{k+1}(s)=\operatorname{argmax}_{a} \hat{Q}_{\theta}^{\pi_{k}}(s, a)$, notably for any $s$ in the dataset. The process iterates until some stopping criterion is satisfied. A classic API algorithm is LSPI (LeastSquares Policy Iteration) [43], which uses LSTD as the value function approximator. However, any other policy evaluation algorithm could be used (TD-Q, GPTD, TDC, etc.). Notice that instead of working with an imposed set of data, new trajectories could be generated at each iteration (generally, using the current estimated policy slightly perturbed, in order to ensure variety of samples).

Approximate value iteration (AVI) consists in computing directly an estimate of the optimal value function. Generally speaking, any value function approximator based on the Bellman optimality operator (such as Q-learning, greedy-GQ, KTD or slLSTD for example) can serve as an AVI algorithm, even if fitted-Q is perhaps best known. Given the available dataset and a value function approximator (based on $T^{*}$ ), an estimate $\hat{Q}_{\theta}^{*}$ of the optimal actionvalue function is computed, and it is used to provide an estimated optimal policy: $\hat{\pi}^{*}(s)=\operatorname{argmax}_{a} \hat{Q}_{\theta}^{*}(s, a)$.

Notice that other approximate dynamic programming algorithms exist, such as ADP based on linear programming [58][59], on modified policy iteration [60] or on conservative policy iteration [61]. However, these do not necessarily rely on a value function approximation algorithm.

\section{B. Online learning}

Assume that the goal is now to learn in an online fashion, that is improving the policy while interacting with the system. This can be handled by an asynchronous API approach: at each time step, the greedy action (with respect to the currently estimated Q-function) is applied and the action-value function is updated using any policy evaluation algorithm (this implies using an online value function approximator, generally in an on-policy setting).

However, learning online induces a dilemma between exploration and exploitation and the greedy action should not be applied at each time step. Instead, from time to time an explorative action (suboptimal according to the currently estimated action-value function but with the potential to improve this estimate) should be chosen. A classic asynchronous API approach is SARSA: the Qfunction is updated according to the TD-Q algorithm (onpolicy setting) and the applied action is chosen according to an $\epsilon$-greedy policy (greedy action with probability $1-\epsilon$, random action else). However, any policy evaluation algorithm could be used instead (such as R-SGD, GPTD, LSTD, etc.), and other action selection schemes could be envisioned (such as a Gibbs sampling approach, for example).

Notice that learning control online induces nonstationarity problems: as the evaluated policy depends on the currently estimated Q-function, it actually changes at each time step, and the value function approximator should take this into account. If gradient-based policy evaluation algorithms handle this well, thanks to the use of a learning rate, this is not the case for least-squaresbased algorithms. A simple solution to this problem is to add a forgetting factor to the minimized cost function:

$$
\theta_{i}=\underset{\omega \in \mathbb{R}^{p}}{\operatorname{argmin}} \sum_{j=1}^{i} \beta^{i-j}\left(\hat{T}_{j} \hat{Q}_{\xi}-\hat{Q}_{\omega}\left(s_{j}, a_{j}\right)\right)^{2},
$$

where $0 \ll \beta<1$ is the forgetting factor. Notice that more general schemes for handling non-stationarity are possible through the original formulation of the KTD framework [11].

Similarly, it is possible to consider asynchronous AVI. This is exactly the same approach, replacing the policy evaluation algorithm by a value function approximator based on the Bellman optimality operator (this approximator must be able to learn in an online fashion, which could exclude fitted-Q). Asynchronous AVI tends to be less cautious than asynchronous API, in the sense that the estimated Q-function do not take into account the explorative steps of the action selection scheme (this cautious aspect also depends heavily on the exploration strategy). This is well illustrated by the cliff-walking task [2, Ch. 6.5].

\section{Actor-critic algorithms}

The online approaches discussed so far use an explicit representation for the action-value function, but not for the policy (which depends explicitly on the Q-function). Actor-critic methods [62][63][64][65][66] use an explicit 
representation for both these objects $(Q$ and $\pi)$. This approach as several advantages. Notably, it allows considering in a principled way continuous actions (which are a problem due to the greedy and the max operators of other approaches) and it somehow implicitly handles the dilemma between exploration and exploitation.

Assume that the policy $\pi_{w}$ is parameterized by some vector $w$. Actor-critic algorithms aim at maximizing the value on a distribution $d_{0}$ of starting state: find $w$ such that $\rho(w)=\mathbb{E}_{s \sim d_{0}}\left[V^{\pi_{w}}\right]$ is maximum. This can be done by performing a gradient ascent (or possibly a natural gradient ascent [65]): $w_{i+1}=w_{i}+\alpha_{i} \nabla \rho\left(w_{i}\right)$. Let $d^{\pi}$ denote the so-called discounted stationary distribution, $d^{\pi}(s)=(1-\gamma) \sum_{i \geq 0} \gamma^{i} P\left(s_{i}=s \mid s_{0}, \pi\right)$, the gradient admits an analytical expression [64]:

$$
\nabla \rho(w)=\frac{1}{1-\gamma} \mathbb{E}_{s \sim d^{\pi} w, a \sim \pi_{w}(. \mid s)}\left[Q^{\pi_{w}}(s, a) \nabla_{w} \pi_{w}(a \mid s)\right] .
$$

Generally speaking, actor-critic approaches maximize $\rho$ using a (natural) gradient ascent, and the gradient $\nabla \rho$ is estimated using an approximation of the Q-function $Q^{\pi_{w}}$ (instead of the unavailable true Q-function).

However, one cannot use any approximation $\hat{Q}_{\theta}$ of $Q^{\pi_{w}}$ [63][64]. First, $\hat{Q}_{\theta}$ must be a "good approximation" of $Q^{\pi_{w}}$ (in a least-squares sense, see [64] for details). Second, the parameterizations of $\hat{Q}_{\theta}$ and $\pi_{w}$ must be "compatible", meaning that they should satisfy [63][64]:

$$
\nabla_{\theta} \hat{Q}_{\theta}(s, a)=\nabla_{w} \ln \pi_{w}(a \mid s) .
$$

Therefore, choosing one parameterization imposes the other.

At this stage, one can remark that if the parameterization is compatible, then $\mathbb{E}_{a \sim \pi(. \mid s)}\left[\hat{Q}_{\theta}(s, a)\right]=0: \hat{Q}_{\theta}$ is not a Q-function, but an advantage function (generally defined as $A(s, a)=Q(s, a)-V(s))$. Algorithms studied in this survey do not allow learning directly such a function, and the literature offers variations of existing algorithms to learn $\hat{Q}_{\theta}(s, a)$ (e.g., see the LSTD-Q variation in [65]).

There is a simple solution to this problem. As shown in [67], it is possible to replace $Q^{\pi_{w}}$ by a "good approximation" $\hat{Q}_{\theta, \xi}(s, a)=f_{\theta}(s, a)+g_{\xi}(s)$ with $\nabla_{\theta} f_{\theta}(s, a)=$ $\nabla_{w} \ln \pi_{w}(a \mid s)$ (no specific condition on $g_{\xi}$ ). This way, $\hat{Q}_{\theta, \xi}$ is actually an action-value function (not an advantage function) and all important theorems of [64][65] can be shown to hold. Therefore, one can plug its favorite parametric policy evaluation algorithm in an actor-critic approach, see [67] for details. However, notice that if the compatibility condition is satisfied, the "good approximation" condition may not be perfectly satisfied. Nevertheless, it often works well in practice.

\section{Algorithms comparison}

This section aims at comparing surveyed algorithms. First, alternative taxonomies are provided. Then, we discuss when choosing what algorithm, depending on the context. Finally, we give some pointers to the literature, regarding experimental results.

\section{A. Alternative taxonomies}

Surveyed algorithms have been divided into three categories (bootstrapping, residual and projected fixed-point) and two subcategories (gradient-based or least-squaresbased). Other taxonomies are possible, as summarized in Tab. II.

First, they either estimate the action-value function of a given policy $\left(Q^{\pi}\right)$ or the optimal Q-function $\left(Q^{*}\right)$ directly. In the first case, they can be used as the policy evaluation component of a more general API scheme, as explained above. In the second case, they provide directly the optimal policy through the estimated Q-function, which is an AVI scheme. This is depicted as pi/vi in Tab. II (equivalently, pi means that the Bellman evaluation operator is considered and vi means that the Bellman optimality operator is considered).

Some of the reviewed algorithms are only defined for linear parameterization of the action-value function, whereas others allow considering nonlinear architectures such as multi-layered neural networks (mainly through a gradient descent or thanks to a statistical linearization). This is depicted as $1 / \mathrm{nl}$ in Tab. II.

A point not discussed so far is the computational (and memory) complexity of the reviewed algorithms. There are basically two cases. For stochastic gradient descentbased algorithms, complexity is in $O(p)$, and for recursive least-squares-based approach, complexity is in $O\left(p^{2}\right)$. Notice that for algorithms handling the Bellman optimality operator, these complexities have to be scaled by the number of actions (because of the max computation). Exceptions (to the two aforementioned cases) are fitted-Q (which complexity depends on the considered supervised learning scheme) and Q-OSP (which is moreover linear in the length of the trajectory). Considering the sample efficiency of these algorithms, second order approaches are usually more efficient (a notable exception being FPKF, see $[68])$.

Lastly, some algorithms produce biased estimates (residual approaches, if not combined with a double sampling scheme or with some colored noise), whereas the other provide unbiased estimates (bootstrapping and projected fixed-point). This is depicted as b/ub in Tab. II. We also recall how $\xi$ is instantiated (see Eq. (5)) and if the algorithm is based on a stochastic gradient descent (sgd) or a recursive least-squares (rls) approach.

\section{B. Which algorithm to choose?}

One can wonder when choosing what algorithm. Unfortunately, there is no easy answer to this. Nevertheless, we try to give some clues here.

First, one may choose between (asynchronous) API and AVI. We think that the choice between an online and a batch learning algorithm (that is asynchronous or not) is more linked to the studied problem than a real choice (is the data imposed? can we sample easily trajectories?). Choosing between API and AVI is a matter of taste (as 


\begin{tabular}{|c|c|c|c|c|c|c|}
\hline & $\begin{array}{l}\text { policy iteration (pi) } \\
\text { value iteration (vi) }\end{array}$ & $\begin{array}{l}\text { linear }(\mathrm{l}) \\
\text { non-linear }(\mathrm{nl})\end{array}$ & $\begin{array}{l}\text { computational } \\
\text { complexity }\end{array}$ & $\begin{array}{c}\text { biased (b) } \\
\text { unbiased (ub) }\end{array}$ & $\begin{array}{c}\xi \\
\text { (see Eq. (5)) }\end{array}$ & $\begin{array}{l}\text { stoch. grad. descent (sgd) } \\
\text { rec. least-squares (rls) }\end{array}$ \\
\hline TD-Q & $\mathrm{pi}$ & $\mathrm{l} / \mathrm{nl}$ & $O(p)$ & $\mathrm{ub}$ & $\theta_{j-1}$ & sgd \\
\hline Q-learning & vi & $\mathrm{l} / \mathrm{nl}$ & $O(p)$ & $\mathrm{ub}$ & $\theta_{j-1}$ & sgd \\
\hline FPKF & $\mathrm{pi} / \mathrm{vi}$ & 1 & $O\left(p^{2}\right)$ & $\mathrm{ub}$ & $\theta_{j-1}$ & rls \\
\hline R-SGD & pi & $\mathrm{l} / \mathrm{nl}$ & $O(p)$ & $\mathrm{b}$ & $\omega$ & sgd \\
\hline GPTD & pi & 1 & $O\left(p^{2}\right)$ & $\mathrm{b}$ & $\omega$ & rls \\
\hline KTD & $\mathrm{pi} / \mathrm{vi}$ & $\mathrm{l} / \mathrm{nl}$ & $O\left(p^{2}\right)$ & $\mathrm{b}$ & $\omega$ & rls \\
\hline GTD2/TDC & pi & $\mathrm{l} / \mathrm{nl}$ & $O(p)$ & $\mathrm{ub}$ & $\theta_{i}$ & sgd \\
\hline Greedy-GQ & vi & 1 & $O(p)$ & $\mathrm{ub}$ & $\theta_{i}$ & sgd \\
\hline LSTD & pi & l & $O\left(p^{2}\right)$ & $\mathrm{ub}$ & $\theta_{i}$ & rls \\
\hline slLSTD & $\mathrm{pi} / \mathrm{vi}$ & $\mathrm{l} / \mathrm{nl}$ & $O\left(p^{2}\right)$ & $\mathrm{ub}$ & $\theta_{i}$ & rls \\
\hline LSPE & pi & l & $O\left(p^{2}\right)$ & $\mathrm{ub}$ & $\theta_{i-1}$ & rls \\
\hline Q-OSP & vi & l & $*$ & $\mathrm{ub}$ & $\theta_{i-1}$ & rls \\
\hline fitted-Q & vi & $\mathrm{l} / \mathrm{nl}$ & ** & $\mathrm{ub}$ & $\theta_{i-1}$ & $* *$ \\
\hline
\end{tabular}

TABLE II

Algorithm Properties.

far as we know, there is no definitive argument in favor of one or the other).

Second, one can choose between a residual and a bootstrapping or a projected fixed-point approach (bootstrapping and projected fixed-point approaches converge to the same limit, when convergence occurs). Residual approaches are more stable and more predictable [69], but they suffer from the bias problem (which disappears when the transitions are deterministic). Other arguments in favor of each approach (residual vs projected fixedpoint) are developed in [70]. Therefore, if the dynamic is (near-) deterministic (or even Lipschitz from our own experience), then a residual method could be chosen (stability argument), else a projected fixed-point or bootstrap approach must be preferred.

If the residual approach is rejected, there is still the choice between bootstrapping and projected fixed-point approaches. Generally speaking, gradient-based bootstrapping algorithms offer less theoretical guarantees than their projected fixed-point counterparts, but they empirically learn faster and tuning meta-parameters is easier. This is no longer true for the least-squares-based algorithms: FPKF is much slower than LSTD (due to the bootstrapping), and they provide the same solution asymptotically.

Then, one can choose between a gradient-based and a least-squares based approach. Least-squares approaches are more sample efficient (which can be theoretically argued in some cases, see for example the finite sample analysis of LSTD [71]), but they also have a higher computational cost. So this choice is a trade-off between sample efficiency and computational cost. If samples are costly to generate or imposed, we advocate to use a leastsquares approach. In the other hand, if there are many features (thus many parameters to be learnt) and if the transitions are easy to generate, gradient-based methods may be preferred.

We have provided a unified derivation of surveyed algorithms, but most of them were introduced differently than in their original derivation. Notably, concerning GPTD [10] and KTD [11], the parameter vector to be learnt is modeled as a random variable of which mean and variance are estimated ${ }^{8}$. Therefore, during learning, uncertainty information about parameters estimates is available. This can be used to compute some variance about the estimated action-value function, for any stateaction couple. This can be useful for solving the dilemma between exploration and exploitation (for example, it may be interesting to choose an action with a low estimated value if this estimate is uncertain or adopt an optimistic action selection). See [41] for a discussion on this subject. The same idea can be adapted to (sl)LSTD, less directly, see [13] for details.

One can also choose between linear and nonliear parameterization. If good linear features are available, it is certainly better to use them, thus to choose a linear parametric value function approximator. However, sometimes a multi-layered artificial neural network can provide better results (generally speaking, the underlying hypothesis space may be richer). In this case, one has to choose an RL algorithm able to handle such nonlinearities. Notice that the only algorithms to come with theoretical guarantees in the nonlinear case are nlTDC and nlGTD2 (but KTD and sILSTD work well in practice, and learn faster, but at a higher computational cost).

Last but not least, the ease of implementation (or the availability of some library) and the ease of choosing the meta-parameters can also drive the choice of the algorithm. Generally speaking, linear-least-squares-based algorithms are easier to tune (there is no learning rate).

\section{Experimental comparisons}

Another point not addressed so far in this article is the thorough experimental comparison of the reviewed algorithms. This is beyond the scope of this algorithmic survey, but Tab. III provides links to the literature, showing where a comparison between algorithm X and algorithm Y can be found (often within a control scheme). This table can be read line by line. Please notice that this does not pretend to be exhaustive (for example, there are some application papers, notably for dialogue management in spoken dialog systems, but not all of them are reported).

\footnotetext{
${ }^{8}$ What allows us deriving it differently here is the close link between recursive least-squares, Kalman filtering and Bayesian filtering.
} 


\begin{tabular}{l|c|c|c|c|c|c|c|c|} 
& gradBoot & FPKF & GPTD & KTD & gradPFP & LSTD & slLSTD & fitted-Q \\
\hline gradBoot & {$[2][27][35]$} & {$[8][68]$} & {$[68]$} & {$[11][40][72]$} & {$[14][15][17]$} & {$[12][43][73][68]$} & {$[13]$} & {$[21]$} \\
\hline FPKF & {$[8][68]$} & & {$[68]$} & & & {$[68]$} & \\
\hline gradRes & {$[9]$} & & & & & & \\
\hline GPTD & {$[68]$} & {$[68]$} & {$[10][25][34][42]$} & {$[11][74]$} & & {$[10][75][68]$} & \\
\hline KTD & {$[11][40][72]$} & & {$[11][74]$} & {$[11][76]$} & & {$[11][40][41][72]$} & {$[13]$} & {$[72]$} \\
\hline gradPFP & {$[14][15][17]$} & & & & {$[14][15]$} & & {$[13]$} & \\
\hline LSTD & {$[12][43][73][68]$} & {$[68]$} & {$[10][75][68]$} & {$[11][40][41][72]$} & & {$[43]$} & {$[13]$} & {$[21][77]$} \\
\hline slLSTD & {$[13]$} & & & {$[13]$} & {$[13]$} & {$[13]$} & & \\
\hline itPFP & {$[54][68]$} & {$[68]$} & {$[68]$} & & & {$[68]$} & & \\
\hline fitted-Q & {$[21]$} & & & {$[72]$} & & {$[21][77]$} & & {$[20][21][51][53]$}
\end{tabular}

TABLE III

Links to EXPERIMENTAL COMPARISONS.

In the table, gradBoot refers to gradient-based bootstrapping algorithms (TD-V, TD-Q, Q-learning), gradRes refers to gradient-based residual algorithms (R-SGD), gradPFP refers to gradient-based projected-fixed-point approaches (GTD2, TDC and greedy-GQ) and itPFP refers to iterated fixed-point algorithms (LSPE and Q-OSP, fitted-Q being left appart). If an algorithm is compared to itself in the table, it means either that it is actually a group of algorithms (as gradBoot for example) or that the paper contains some illustrative example (as the illustration of the variance information provided by the GPTD algorithm in [10]) or some problem-specific experiment/large-scale application.

\section{OTHER VALUE FUNCTION APPROXIMATORS}

This article surveys parametric value function approximators, but there exist other ways to estimate a value function. Some of them are briefly mentioned hereafter.

\section{A. Extension to eligibility traces}

TD learning with eligibility traces [2], known as $\operatorname{TD}(\lambda)$, provides a nice bridge between temporal difference learning and Monte Carlo learning, and by controlling the bias/variance trade-off [78], their use can significantly speed up learning. When the value function is approximated through a linear architecture, the depth $\lambda$ of the eligibility traces is also known to control the quality of approximation [26].

Using eligibility traces amounts to looking for the fixedpoint of the following variation of the Bellman operator [1] (here we assume a fixed policy, that is the evaluation problem): $T^{\lambda} V=(1-\lambda) \sum_{i=0}^{\infty} \lambda^{i} T^{i+1} V$. As we have introduced a sampled Bellman operator to derive surveyed algorithms, the same can be done for this $T^{\lambda}$ operator. For a partial trajectory $\left(s_{k}, r_{k}, s_{k+1}\right)_{j \leq k \leq i-1}$, we define $\hat{T}_{j, i}^{\lambda}$ the sampled and truncated operator (e.g., see [68]):

$$
\hat{T}_{j, i}^{\lambda} V=V\left(s_{j}\right)+\sum_{k=j}^{i}(\gamma \lambda)^{k-j}\left(r_{k}+\gamma V\left(s_{k+1}\right)-V\left(s_{k}\right)\right) .
$$

This being defined, the work done in this survey can be repeated by replacing $\hat{T}_{j}$ by $\hat{T}_{j, i}^{\lambda}$ in Eq. (5):

$$
\theta_{i}=\underset{\omega \in \mathbb{R}^{p}}{\operatorname{argmin}} \sum_{j=1}^{i}\left(\hat{T}_{j, i}^{\lambda} \hat{V}_{\xi}-\hat{V}_{\omega}\left(s_{j}, a_{j}\right)\right)^{2}
$$

Notice that this only holds for the evaluation problem (the Bellman optimality operator is not considered) and in the on-policy case. To handle the off-policy case, it is necessary to use also importance sampling. See [68][79], where this approach is used to derive new off-policy least-squaresbased and eligibility-traces-based algorithms.

\section{B. Nonparametric approaches}

Given an approximation architecture, this survey has shown that there exists a bunch of approaches to learn the corresponding parameters. However, choosing a judicious hypothesis space is highly problem-dependent and it is generally a difficult issue. Therefore, we think that research fields such as feature construction, feature selection, or more generally non-parametric value function approximation are of primary importance.

The GPTD algorithm [25] is kernel-based and nonparametric in its more general form (not presented here). Using the same idea (that is constructing the span of the feature space implicitly defined by a Mercer kernel), the LSTD algorithm has been kernelized [80] as well as the LSPE algorithm [81]. More generally, kernelized approaches to value function approximation are discussed in $[82]$.

Feature selection via $\ell_{1}$-regularization has also been envisioned for non-parametric value function approximation: adding an $\ell_{1}$-penalty term imposes some parameters to be exactly zero, which allows handling the case $p \gg n$ (much more features than samples). Adding such a penalty term to value function approximation is not straightforward and there are many ways to do it. See [83] for an approach based on residual minimization and [84][85][86][87][88] for approaches based on projected fixed-point ([88] provides a synthetic overview of these methods).

Another approach consists in generating features, based on the Bellman error [89][90][91][92][93] or upon graph structure built from observed trajectories through the state space [94]. State aggregation (e.g., [95][96]) can also be seen as a feature generation scheme. Other methods are based on local averagers or kernel smoothers (usually Nadaraya-Watson-like estimators) [52][97][98]. These are examples among others, this general topic would deserve another survey. 


\section{Other criteria}

In this article, the quality of a policy is quantified by the value function defined as the expected discounted cumulative reward, $V^{\pi}(s)=\mathbb{E}\left[\sum_{i>0} \gamma^{i} r_{i} \mid s_{0}=s, \pi\right]$. Other criteria can be envisioned, such as the finite horizon criterion (and the value function is then $V^{\pi}(s)=$ $\mathbb{E}\left[\sum_{i=0}^{H} r_{i} \mid s_{0}=s, \pi\right]$ for some predefined horizon $\left.H\right)$ or the average-reward criterion (for which the value function is defined as $\left.V^{\pi}(s)=\lim _{H \rightarrow \infty} \frac{1}{H} \mathbb{E}\left[\sum_{i=0}^{H} r_{i} \mid s_{0}=s, \pi\right]\right)$. The $\gamma$-discounted criterion considered in this article is probably the most frequently used in the literature, but notice that the work done here cannot be directly adapted to the other criteria.

\section{Conclusion}

This article has reviewed a large part of the state of the art in parametric (action-) value function approximation. Basically, it has been shown that all these approaches can be derived from the unified cost function (5). Further, they can be categorized in three main classes, given the considered cost function (related to bootstrapping, residual or projected fixed-point). In each of these groups, they can be categorized given that the cost function is minimized using a stochastic gradient descent or a recursive least-squares approach (except fitted-Q, which can be considered with any supervised learning algorithm). Projected fixed-point approaches can be divided into two approaches, given that the cost function is directly minimized or that the underlying possible fixed-point is searched for using an iterative scheme. All of this is summarized in Tab. I on Page 5. Sec. VI to VIII have given clues on how the surveyed value function approximators can be used for control, how they can be compared and what are other possible (not covered) approaches for estimating a value function.

\section{ACKnowledgments}

The authors would like to thank Jérémy Fix and Bruno Scherrer for proofreading earlier versions of this article, as well as the anonymous reviewers for useful comments and suggestions. This research was partly funded by the EU FP7 FET project ILHAIRE (grant $n^{\circ} 270780$ ) and the Région Lorraine (France).

\section{REFERENCES}

[1] D. P. Bertsekas and J. N. Tsitsiklis, Neuro-Dynamic Programming (Optimization and Neural Computation Series, 3). Athena Scientific, 1996.

[2] R. S. Sutton and A. G. Barto, Reinforcement Learning: An Introduction, 3rd ed. The MIT Press, March 1998.

[3] O. Sigaud and O. Buffet, Eds., Markov Decision Processes and Artificial Intelligence. Wiley - ISTE, 2010.

[4] C. Szepesvári, Algorithms for Reinforcement Learning. Morgan and Claypool, 2010.

[5] M. Wiering and M. van Otterlo, Reinforcement Learning: State Of the Art. Springer, 2012.

[6] L. Busoniu, R. Babuška, B. De Schutter, and D. Ernst, Reinforcement Learning and Dynamic Programming Using Function Approximators. CRC Press, 2010.

[7] W. Powell, Approximate Dynamic Programming: Solving the Curses of Dimensionality. Wiley, 2007.
[8] D. Choi and B. Van Roy, "A Generalized Kalman Filter for Fixed Point Approximation and Efficient Temporal-Difference Learning," Discrete Event Dynamic Systems, vol. 16, pp. 207239, 2006.

[9] L. C. Baird, "Residual Algorithms: Reinforcement Learning with Function Approximation," in International Conference on Machine Learning (ICML), 1995, pp. 30-37.

[10] Y. Engel, "Algorithms and Representations for Reinforcement Learning," Ph.D. dissertation, Hebrew University, April 2005.

[11] M. Geist and O. Pietquin, "Kalman Temporal Differences," Journal of Artificial Intelligence Research (JAIR), 2010.

[12] S. J. Bradtke and A. G. Barto, "Linear Least-Squares algorithms for temporal difference learning," Machine Learning, vol. 22, no. 1-3, pp. 33-57, 1996.

[13] M. Geist and O. Pietquin, "Statistically Linearized LeastSquares Temporal Differences," in IEEE International Conference on Ultra Modern Control Systems (ICUMT). Moscow (Russia): IEEE, October 2010.

[14] R. S. Sutton, H. R. Maei, D. Precup, S. Bhatnagar, D. Silver, C. Szepesvári, and E. Wiewiora, "Fast gradient-descent methods for temporal-difference learning with linear function approximation," in International Conference on Machine Learning (ICML). New York, NY, USA: ACM, 2009, pp. 993-1000.

[15] H. Maei, C. Szepesvari, S. Bhatnagar, D. Precup, D. Silver, and R. S. Sutton, "Convergent temporal-difference learning with arbitrary smooth function approximation," in Advances in Neural Information Processing Systems (NIPS), Y. Bengio, D. Schuurmans, J. Lafferty, C. K. I. Williams, and A. Culotta, Eds., 2009, pp. 1204-1212.

[16] H. R. Maei and R. S. Sutton, "GQ $(\lambda)$ : a general gradient algorithm for temporal-differences prediction learning with eligibility traces," in Conference on Artificial General Intelligence, 2010.

[17] H. R. Maei, C. Szepesvari, S. Bhatnagar, and R. S. Sutton, "Toward Off-Policy Learning Control with Function Approximation," in International Conference on Machine Learning (ICML), 2010.

[18] A. Nedić and D. P. Bertsekas, "Least Squares Policy Evaluation Algorithms with Linear Function Approximation," Discrete Event Dynamic Systems: Theory and Applications, vol. 13, pp. 79-110, 2003.

[19] H. Yu and D. P. Bertsekas, "Q-Learning Algorithms for Optimal Stopping Based on Least Squares," in European Control Conference, Kos, Greece, 2007.

[20] G. Gordon, "Stable Function Approximation in Dynamic Programming," in International Conference on Machine Learning (IMCL), 1995.

[21] D. Ernst, P. Geurts, and L. Wehenkel, "Tree-Based Batch Mode Reinforcement Learning," Journal of Machine Learning Research, vol. 6, pp. 503-556, 2005.

[22] R. S. Sutton, "Learning to predict by the methods of temporal differences," Machine Learning, vol. 3, no. 1, pp. 9-44, 1988.

[23] G. A. Rummery and M. Niranjan, "Online q-learning using connectionist systems," Cambridge University, Tech. Rep. CUED/F-INFENG/TR 166, 1994.

[24] C. J. Watkins and P. Dayan, "Q-learning," Machine Learning, vol. 8, pp. 279-292, 1992.

[25] Y. Engel, S. Mannor, and R. Meir, "Bayes Meets Bellman: The Gaussian Process Approach to Temporal Difference Learning," in International Conference on Machine Learning (ICML), 2003, pp. $154-161$.

[26] J. N. Tsitsiklis and B. Van Roy, "An analysis of temporaldifference learning with function approximation," IEEE Transactions on Automatic Control, vol. 42, 1997.

[27] G. Tesauro, "Temporal Difference Learning and TD-Gammon," Communications of the ACM, vol. 38, no. 3, March 1995.

[28] H. van Seijen, H. van Hasselt, S. Whiteson, and M. Wiering, "A Theoretical and Empirical Analysis of Expected Sarsa," in IEEE International Symposium on Adaptive Dynamic Programming and Reinforcement Learning (ADPRL), 2009.

[29] H. Yu, "Least Squares Temporal Difference Methods: An Analysis Under General Conditions," University of Helsinki, Tech. report C-2010-39, 2010.

[30] F. S. Melo, S. P. Meyn, and M. I. Ribeiro, "An analysis of reinforcement learning with function approximation," in International Conference on Machine Learning (ICML), 2009, pp. 664-671. 
[31] A. Antos, C. Szepesvári, and R. Munos, "Learning near-optimal policies with Bellman-residual minimization based fitted policy iteration and a single sample path," Machine Learning, vol. 71, no. 1, pp. 89-129, 2008.

[32] A. Kruger, "On Fréchet subdifferentials," Journal of Mathematical Sciences, vol. 116, pp. 3325-3358, 2003.

[33] T. W. Anderson, An Introduction to Multivariate Statistical Analysis. Wiley, 1984.

[34] Y. Engel, S. Mannor, and R. Meir, "Reinforcement Learning with Gaussian Processes," in International Conference on Machine Learning (ICML), 2005.

[35] D. Precup, R. S. Sutton, and S. P. Singh, "Eligibility Traces for Off-Policy Policy Evaluation," in International Conference on Machine Learning (ICML). San Francisco, CA, USA: Morgan Kaufmann Publishers Inc., 2000, pp. 759-766.

[36] S. J. Julier and J. K. Uhlmann, "A new extension of the Kalman filter to nonlinear systems," in Int. Symp. Aerospace/Defense Sensing, Simul. and Controls 3, 1997.

[37] S. J. Julier, "The scaled unscented transformation," in American Control Conference, vol. 6, 2002, pp. 4555-4559.

[38] P. M. Nørgård, N. Poulsen, and O. Ravn, "New developments in state estimation for nonlinear systems," Automatica, vol. 36, no. 11, pp. 1627-1638, 2000.

[39] R. van der Merwe, "Sigma-point kalman filters for probabilistic inference in dynamic state-space models," Ph.D. dissertation, OGI School of Science \& Engineering, Oregon Health \& Science University, Portland, OR, USA, April 2004.

[40] M. Geist and O. Pietquin, "Eligibility Traces through Colored Noises," in IEEE International Conference on Ultra Modern Control Systems (ICUMT). Moscow (Russia): IEEE, October 2010.

[41] _ "Managing Uncertainty within the KTD framework," in Active Learning and Experimental Design Workshop (collocated with AISTATS 2010), ser. Journal of Machine Learning Research - Workshop and Conference Proceedings, Sardinia, Italy, 2010.

[42] L. Daubigney, M. Gasic, S. Chandramohan, M. Geist, O. Pietquin, and S. Young, "Uncertainty management for online optimisation of a POMDP-based large-scale spoken dialogue system," in Annual Conference of the International Speech Communication Association (Interspeech), Florence (Italy), August 2011, pp. 1301-1304.

[43] M. G. Lagoudakis and R. Parr, "Least-squares policy iteration," Journal of Machine Learning Research, vol. 4, pp. 1107-1149, 2003.

[44] T. Söderström and P. Stoica, "Instrumental variable methods for system identification," Circuits, Systems, and Signal Processing, vol. 21, pp. 1-9, 2002.

[45] A. Geramifard, M. Bowling, and R. S. Sutton, "Incremental Least-Squares Temporal Difference Learning," in Conference of American Association for Artificial Intelligence (AAAI), 2006, pp. 356-361.

[46] J. Johns, M. Petrik, and S. Mahadevan, "Hybrid least-squares algorithms for approximate policy evaluation," Machine learning, 2009.

[47] M. Geist and O. Pietquin, "Statistically Linearized Recursive Least Squares," in IEEE International Workshop on Machine Learning for Signal Processing (MLSP). Kittilä (Finland): IEEE, 2010

[48] R. S. Sutton, C. Szepesvari, and H. R. Maei, "A Convergent $\mathrm{O}(\mathrm{n})$ Algorithm for Off-policy Temporal-difference Learning with Linear Function Approximation," in Advances in Neural Information Processing Systems (NIPS), Vancouver, BC, 2008.

[49] B. D. Ripley, Stochastic Simulation. Wiley \& Sons, 1987.

[50] A. Samuel, "Some studies in machine learning using the game of checkers," IBM Journal on Research and Development, pp. 210-229, 1959.

[51] R. Munos, "Performance Bounds in Lp norm for Approximate Value Iteration," SIAM Journal on Control and Optimization, 2007.

[52] D. Ormoneit and S. Sen, "Kernel-Based Reinforcement Learning," Machine Learning, vol. 49, pp. 161-178, 2002.

[53] M. Riedmiller, "Neural Fitted Q Iteration - First Experiences with a Data Efficient Neural Reinforcement Learning Method ," in European Conference on Machine Learning (ECML), 2005.
[54] D. P. Bertsekas and S. Ioffe, "Temporal Differences-Based Policy Iteration and Applications in Neuro-Dynamic Programming," Labs for Information and Decision Systems, MIT, Tech. Rep. LIDS-P-2349, 1996

[55] D. P. Bertsekas, V. Borkar, and A. Nedic, Learning and Approximate Dynamic Programming. IEEE Press, 2004, ch Improved Temporal Difference Methods with Linear Function Approximation, pp. 231-235.

[56] D. P. Bertsekas and H. Yu, "Projected Equation Methods for Approximate Solution of Large Linear Systems," Journal of Computational and Applied Mathematics, vol. 227, pp. 27-50, 2007.

[57] D. P. Bertsekas, "Projected Equations, Variational Inequalities, and Temporal Difference Methods," in IEEE International Symposium on Adaptive Dynamic Programming and Reinforcement Learning (ADPRL), 2009.

[58] D. P. de Farias and B. V. Roy, "The Linear Programming Approach to Approximate Dynamic Programming," Operations Research, vol. 51, no. 6, pp. 850-865, 2003.

[59] V. V. Desai, V. F. Farias, and C. C. Moallemi, "The Smoothed Approximate Linear Program," in Advances in Neural Information Processing Systems (NIPS), 2009.

[60] B. Scherrer, V. Gabillon, M. Ghavamzadeh, and M. Geist, "Approximate Modified Policy Iteration," in International Conference on Machine Learning (ICML), 2012.

[61] S. Kakade and J. Langford, "Approximately optimal approximate reinforcement learning," in International Conference on Machine Learning (ICML), 2002.

[62] A. Barto, R. Sutton, and C. Anderson, "Neuronlike adaptive elements that can solve difficult learning control problems," IEEE Transactions on System, Man, and Cybernetics, vol. 13, pp. 834-846, 1983.

[63] V. R. Konda and J. N. Tsitsiklis, "On Actor-Critic Algorithms," SIAM Journal on Control and Optimization, vol. 42, no. 4, pp. 1143-1166, 2003.

[64] R. S. Sutton, D. A. McAllester, S. P. Singh, and Y. Mansour, "Policy Gradient Methods for Reinforcement Learning with Function Approximation," in Neural Information Processing Systems (NIPS), 1999, pp. 1057-1063.

[65] J. Peters and S. Schaal, "Natural Actor-Critic," Neurocomputing, vol. 71, pp. 1180-1190, 2008.

[66] S. Bhatnagar, R. S. Sutton, M. Ghavamzadeh, and M. Lee, "Incremental natural actor-critic algorithms," in Advances in Neural Information Processing Systems (NIPS), Vancouver, Canada, 2007.

[67] M. Geist and O. Pietquin, "Revisiting natural actor-critics with value function approximation," in International Conference on Modeling Decisions for Artificial Intelligence (MDAI), ser. LNAI, vol. 6408. Modeling Decisions for Artificial Intelligence, 2010, pp. 207-218.

[68] B. Scherrer and M. Geist, "Recursive Least-Squares Learning with Eligibility Traces," in European Workshop on Reinforcement Learning (EWRL 2011), 2011.

[69] R. Munos, "Error Bounds for Approximate Policy Iteration," in International Conference on Machine Learning (ICML), 2003, pp. 560-567.

[70] B. Scherrer, "Should one compute the Temporal Difference fix point or minimize the Bellman Residual? The unified oblique projection view," in International Conference on Machine Learning (ICML), 2010.

[71] A. Lazaric, M. Ghavamzadeh, and R. Munos, "Finite-Sample Analysis of LSTD," in International Conference on Machine Learning (ICML), 2010.

[72] O. Pietquin, M. Geist, and S. Chandramohan, "Sample Efficient On-line Learning of Optimal Dialogue Policies with Kalman Temporal Differences," in International Joint Conference on Artificial Intelligence (IJCAI), Barcelona, Spain, July 2011, pp. 1878-1883.

[73] J. A. Boyan, "Technical Update: Least-Squares Temporal Difference Learning," Machine Learning, vol. 49, no. 2-3, pp. 233-246, 1999

[74] L. Daubigney, M. Geist, and O. Pietquin, "Off-policy Learning in Large-scale POMDP-based Dialogue Systems," in IEEE International Conference on Acoustics, Speech and Signal Processing (ICASSP). Kyoto (Japan): IEEE, 2012. 
[75] C. W. Phua and R. Fitch, "Tracking value function dynamics to improve reinforcement learning with piecewise linear function approximation," in International Conference on Machine Learning (ICML), 2007.

[76] M. Keramati, A. Dezfouli, and P. Piray, "Speed/Accuracy Trade-Off between the Habitual and the Goal-Directed Processes," PLoS Comput Biol, vol. 7, 052011.

[77] O. Pietquin, M. Geist, S. Chandramohan, and H. Frezza-Buet, "Sample-Efficient Batch Reinforcement Learning for Dialogue Management Optimization," ACM Transactions on Speech and Language Processing, vol. 7, no. 3, pp. 7:1-7:21, 2011.

[78] M. Kearns and S. Singh, "Bias-Variance Error Bounds for Temporal Difference Updates," in Conference on Learning Theory (COLT), 2000.

[79] B. Scherrer and M. Geist, "Recursive least-squares off-policy learning with eligibility traces," INRIA, Tech. Rep., 2012.

[80] X. Xu, D. Hu, and X. Lu, "Kernel-Based Least Squares Policy Iteration for Reinforcement Learning," IEEE Transactions on Neural Networks, vol. 18, no. 4, pp. 973-992, July 2007.

[81] T. Jung and D. Polani, "Kernelizing LSPE $(\lambda)$," in IEEE Symposium on Approximate Dynamic Programming and Reinforcement Learning (ADPRL), 2007, pp. 338-345.

[82] G. Taylor, , and R. Parr, "Kernelized Value Function Approximation for Reinforcement Learning," in International Conference on Machine Learning (ICML), 2009.

[83] M. Loth, M. Davy, and P. Preux, "Sparse temporal difference learning using lasso," in IEEE International Symposium on Approximate Dynamic Programming and Reinforcement Learning (ADPRL), Hawaï, USA, April 2007.

[84] J. Z. Kolter and A. Y. Ng, "Regularization and Feature Selection in Least-Squares Temporal Difference Learning," in International Conference on Machine Learning (ICML), Montreal Canada, 2009

[85] J. Johns, C. Painter-Wakefield, and R. Parr, "Linear Complementarity for Regularized Policy Evaluation and Improvement," in Advances in Neural Information Processing Systems (NIPS), J. Lafferty, C. K. I. Williams, J. Shawe-Taylor, R. Zemel, and A. Culotta, Eds., 2010, pp. 1009-1017.

[86] M. Geist and B. Scherrer, " $\ell_{1}$-penalized projected Bellman residual," in European Workshop on Reinforcement Learning (EWRL), 2011

[87] M. W. Hoffman, A. Lazaric, M. Ghavamzadeh, and R. Munos, "Regularized Least Squares Temporal Difference learning with nested $\ell_{2}$ and $\ell_{1}$ penalization," in European Workshop on Reinforcement Learning (EWRL), 2011.

[88] M. Geist, B. Scherrer, A. Lazaric, and M. Ghavamzadeh, "A Dantzig Selector Approach to Temporal Difference Learning," in International Conference on Machine Learning (ICML), 2012.

[89] I. Menache, S. Mannor, and N. Shimkin, "Basis Function Adaptation in Temporal Difference Reinforcement Learning," Annals of Operations Research, vol. 134, no. 1, pp. 215-238, 2005.

[90] P. W. Keller, S. Mannor, and D. Precup, "Automatic basis function construction for approximate dynamic programming and reinforcement learning," in International Conference on Machine Learning (ICML). New York, NY, USA: ACM Press, 2006, pp. 449-456.

[91] R. Parr, C. Painter-Wakefield, L. Li, and M. Littman, "Analyzing Feature Generation for Value-Function Approximation," in International Conference on Machine Learning (ICML), 2007.

[92] R. Parr, L. Li, G. Taylor, C. Painter-Wakefield, and M. L. Littman, "An Analysis of Linear Models, Linear ValueFunction Approximation, and Feature Selection for Reinforcement Learning," in International Conference on Machine Learning (ICML), Helsinki, Finland, 2008.

[93] J. Wu and R. Givan, "Automatic Induction of Bellman-Error Features for Probabilistic Planning," Journal of Artificial Intelligence Research (JAIR), 2010.

[94] S. Mahadevan and M. Maggioni, "Proto-value Functions: A Laplacian Framework for Learning Representation and Control in Markov Decision Processes ," Journal of Machine Learning Research, vol. 8, pp. 2169-2231, 2007.

[95] D. Bertsekas and D. Castañon, "Adaptive aggregation methods for infinite horizon dynamic programming," IEEE Transactions on Automatic Control, vol. 34, no. 6, pp. 589-598, 1989.

[96] S. Singh, T. Jaakkola, and M. Jordan, "Reinforcement learning with soft state aggregation," Advances in neural information processing systems (NIPS), pp. 361-368, 1995
[97] J. Ma and W. B. Powell, "Convergence Analysis of Kernel-based On-policy Approximate Policy Iteration Algorithms for Markov Decision Processes with Continuous, Multidimensional States and Actions," Princeton University, Tech. Rep., 2010.

[98] A. Barreto, D. Precup, and J. Pineau, "Reinforcement learning using kernel-based stochastic factorization," in Advances in Neural Information Processing Systems (NIPS), 2011.

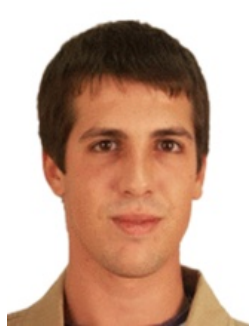

Matthieu Geist obtained an Electrical Engineering degree and an Msc degree in Mathematics from Supélec (France), both in September 2006, as well as a PhD degree in Mathematics from the "Université Paul Verlaine de Metz" (France) in November 2009. From January 2007 to January 2010, he was a member of the Measure and Control lab (MC cluster) of ArcelorMittal Research and a member of the CORIDA project-team of INRIA. In February 2010, he joined the IMS-MaLIS research group of Supélec as an assistant professor. His research interests include statistical machine learning (especially reinforcement learning), as well as applications to spoken dialogue systems. He authored or coauthored more than 50 publications in these fields.

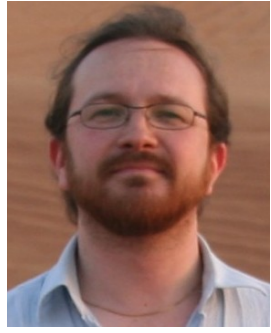

Olivier Pietquin (M'01 - SM'11) obtained an Electrical Engineering degree from the Faculty of Engineering, Mons (FPMs, Belgium) in June 1999 and a PhD degree in April 2004. In 2011, he received the Habilitation à Diriger des Recherches (French Tenure) from the University Paul Sabatier (Toulouse, France). He joined the FPMs Signal Processing department (TCTS Lab.) in September 1999. In 2001, he has been a visiting researcher at the Speech and Hearing lab of the University of Sheffield (UK). Between 2004 and 2005, he was a Marie-Curie Fellow at the Philips Research lab in Aachen (Germany). Now he is a Professor at the Metz campus of the Ecole Supérieure d'Electricité (Supélec, France). He is also a full member of the UMI 2958 (joint lab with GeorgiaTech and CNRS) since 2010 where he coordinates the computer science departments and heads the Machine Learning and Interactive Systems group. From 2007 to 2011, he was also a member of the IADI INSERM research team (in biomedical signal processing). Since 2010, Olivier Pietquin sits at the IEEE Speech and Language Technical Committee. His research interests include spoken dialog systems evaluation, simulation and automatic optimisation, machine learning, speech and signal processing. He authored or co-authored more than 100 publications in these domains. 